\title{
Efficient Multiexciton State Generation in Charge-Transfer Coupled Perylene Bisimide Dimers via Structural Control
}

\author{
Yongseok Hong ${ }^{\dagger}$, Juno Kim ${ }^{\dagger}$, Woojae $\mathrm{Kim}^{\dagger}$, Christina Kaufmann ${ }^{\ddagger}$, Hyungjun Kim${ }^{\S, \perp *}$, Frank Würthner ${ }^{\ddagger *}$ and
} Dongho $\mathrm{Kim}^{+*}$

\section{Supplementary Information}

1. Experimental methods

2. Steady state absorption \& emission

3. fs-Transient absorption data

4. ns-Transient absorption data

5. TCSPC data

6. Broadband fluorescence up-conversion data

7. Quantum chemical simulations

8. References 


\section{Experimental methods}

\section{Sample preparation and steady-state measurements:}

PBI monomer, Bis-PBI N (N=1,2 and 3) were prepared according to the reported methods. ${ }^{1}$ Steady-state absorption spectra were measured on a UV/Vis/NIR spectrometer (Varian, Cary5000) and fluorescence spectra were measured on a fluorescence spectrophotometer (Hitachi, F-7000). Fluorescence spectra are spectrally corrected by using correction factor of the fluorescence spectrophotometer. HPLC-grade solvents were purchased from Sigma-Aldrich and used without further purification. All measurements are measured in Ar saturated condition.

\section{Time-correlated single photon counting (TCSPC)}

A time-correlated single-photon-counting (TCSPC) system was used for measurements of spontaneous fluorescence decay. As an excitation light source, we used a mode-locked Ti:sapphire oscillator (Spectra Physics, MaiTai BB) which provides ultrashort pulse (center wavelength of $800 \mathrm{~nm}$ with $80 \mathrm{fs}$ at FWHM) with high repetition rate $(80 \mathrm{MHz})$. This high repetition rate was reduced to $800 \mathrm{kHz}$ by using homemade pulse-picker. The pulse-picked output was frequency doubled by a 1 -mm-thick BBO crystal (type-I, $\theta=29.2^{\circ}$, EKSMA). The fluorescence was collected by a microchannel plate photomultiplier (MCP-PMT, Hamamatsu, R3809U-51) with a thermoelectric cooler (Hamamatsu, C4878) connected to a TCSPC board (Becker \& Hickel SPC-130). The overall instrumental response function was about 25 ps (FWHM). A vertically polarized pump pulse by a Glanlaser polarizer was irradiated to samples, and a sheet polarizer set at an angle complementary to the magic angle $\left(54.7^{\circ}\right)$, was placed in the fluorescence collection path to obtain polarization-independent fluorescence decays.

\section{Femtosecond broadband fluorescence upconversion spectroscopy (FLUPS)}

Femtosecond broadband fluorescence up-conversion (FLUPS) apparatus ${ }^{2-6}$ was used for obtaining the transient fluorescence spectra. A Ti:sapphire laser system (Spectra-Physics, Spitfire) provides $35 \mathrm{fs,} 380 \mu \mathrm{J}$ pulses at 800 $\mathrm{nm}$ with $10 \mathrm{kHz}$ repetition rate. The output beam is divided by beam splitter with the equivalent ratio. A pulse of $190 \mu \mathrm{J}$ is used to pump a S3 commercial collinear optical parametric amplifier (TOPAS, Light Conversion) which delivers $50 \mathrm{fs}, \sim 20 \mu \mathrm{J}$ gate pulses $(\sim 1300 \mathrm{~nm})$ with the vertical polarization. The gate beam passes through a periscope, which adjusts the height and rotates the vertically polarized pulse to be horizontally polarized. Then, the gate pulse passes a sequence of SF50 prism $\left(55.5^{\circ}\right)$ compressor with optimal separation of $12 \mathrm{~cm}$ between the apexes of each prism. Finally, the gate beam is relayed onto the nonlinear crystal by a lens ( $\mathrm{f}=100 \mathrm{~mm}, \mathrm{Tc}=2$ $\mathrm{mm}$ ). The pulse energy of the gate beam is attenuated by the neutral density filter to keep a level below $6 \mu \mathrm{J}$. The rest of the fundamental light is used as a source for the tunable homemade optical parametric amplifiers (OPA) system. This homemade OPA system is based on noncollinear phase-matching geometry in a nonlinear crystal (EKSMA, BBO, $\theta=32^{\circ}, \varphi=0^{\circ}, \mathrm{d}=2 \mathrm{~mm}$ ), which is easily color-tuned by controlling optical delay between white light continuum seed pulses $(450-1400 \mathrm{~nm})$ produced by using a sapphire window $(\mathrm{d}=2 \mathrm{~mm})$ and visible pump pulses $(400 \mathrm{~nm})$ generated by a frequency doubling nonlinear crystal (EKSMA, BBO, $\theta=29.2^{\circ}$ ). The generated visible OPA pulses had a pulse width of $\sim 20 \mathrm{fs}$ and an average power of $20 \mathrm{~mW}$ at $10 \mathrm{kHz}$ repetition rate in the range of 480-700 $\mathrm{nm}$ with vertical polarization. In order to adjust the pulse polarization to prism compressors, the pulse passes through the periscope and then passes through a fused-silica prism compressor $\left(69^{\circ}\right)$, which has the optimal separation of $95 \mathrm{~cm}$. In order to prevent polarization-dependent signals, the pulse polarization is controlled with a half wave plate to be a magic angle $\left(54.7^{\circ}\right)$ and finally the beam is focused onto a $500 \mu \mathrm{m}$ thick quartz cuvette containing sample with a lens $(\mathrm{f}=300 \mathrm{~mm}, \mathrm{Tc}=2 \mathrm{~mm})$. The pulse energy is attenuated by the ND filter to keep a level below $60 \mathrm{~nJ}$. Moreover, the cuvette is mounted on a motor-driven stage and continuously moved back and forth to avoid photo-degradation and the thermal lens effect. Collection of the fluorescence is achieved by a reflecting microscope objective lens (Newport). Finally, the collected fluorescence is relayed onto the nonlinear crystal by the off-axis parabolic mirror (Newport, $\mathrm{f}=50 \mathrm{~mm}$ ). The horizontally polarized extraordinary upconverted signal is emitted from the BBO crystal (EKSMA, $\theta=40^{\circ}, \varphi=0^{\circ}, \mathrm{d} \mathrm{S} 4=0.5$ $\mathrm{mm}$ ) with type II interaction between fluorescence (o) and gate (e) pulses based on noncollinear geometry (an angle between fluorescence and gate pulses, $\alpha=\sim 25^{\circ}$ ). Unwanted light of horizontal polarization, stemming from 
the original fluorescence and the pump pulse (or Rayleigh scattered light), is mostly ejected by a wire-grid polarizer (Moxtec PPL04C). Moreover, the upconverted signals pass a Glan-Taylor calcite polarizer (Thorlabs, GT10-A) in order to eliminate unwanted light of vertical polarization, originating from the remaining original fluorescence. The upconverted signals are imaged dispersion-free onto the entrance slit of a spectrograph (Princeton Instrument, Isoplane ${ }^{\circledR}$ SCT 320) and then the upconverted spectrum is finally registered with a CCD camera (Andor Technology, DV420 BU). The FWHM of the cross-correlation functions between the scattered pump pulse (i.e., $520 \mathrm{~nm}$ ) and the gate pulse (i.e., $1300 \mathrm{~nm}$ ) is measured to be $170 \mathrm{fs}$. The transient fluorescence spectrum at each time delay was recorded with $1 \mathrm{~s} \mathrm{CCD} \mathrm{exposure} \mathrm{time} \mathrm{and} \mathrm{was} \mathrm{averaged} \mathrm{by} \mathrm{using} 10$ successively recorded spectra. The chirp due to group velocity dispersion (GVD) was measured based on the instantaneous response of DCM dye in toluene. Photometric correction (from 420 to $850 \mathrm{~nm}$ ) was applied by using standard dye solutions (1) DCM in toluene, 2) DCM in methanol, and 3) LDS751 in methanol as described in the literature. 4

\section{Femtosecond transient absorption spectroscopy}

The femtosecond time-resolved transient absorption (fs-TA) spectrometer consists of an optical parametric amplifier (OPA; Palitra, Quantronix) pumped by a Ti:sapphire regenerative amplifier system (Integra-C, Quantronix) operating at $1 \mathrm{kHz}$ repetition rate and an optical detection system. The generated OPA pulses have a pulse width of $\sim 100 \mathrm{fs}$ in the range of $280-2700 \mathrm{~nm}$, which are used as pump pulses. White light continuum (WLC) probe pulses were generated using a sapphire window (4 $\mathrm{mm}$ thick) by focusing a small portion of the fundamental $800 \mathrm{~nm}$ pulses which was picked off by a quartz plate before entering the OPA. The time delay between pump and probe beams was carefully controlled by making the pump beam travel along a variable optical delay (ILS250, Newport). Intensities of the spectrally dispersed WLC probe pulses are monitored by a High S5 Speed Spectrometer (Ultrafast Systems) for both visible and near-infrared measurements. To obtain the timeresolved transient absorption difference signal $(\Delta \mathrm{A})$ at a specific time, the pump pulses were chopped at $500 \mathrm{~Hz}$ and absorption spectra intensities were saved alternately with or without pump pulse. Typically, 4000 pulses excite the samples to obtain the fs-TA spectra at each delay time. The polarization angle between pump and probe beam was set at the magic angle $\left(54.7^{\circ}\right)$ using a Glan-laser polarizer with a half-wave retarder in order to prevent polarization-dependent signals. Cross-correlation fwhm in pump-probe experiments was around $200 \mathrm{fs}$ and chirp of WLC probe pulses was measured to be $1.2 \mathrm{ps}$ in the $450-800 \mathrm{~nm}$ region. To minimize chirp, all reflection optics were used in the probe beam path. A quartz cell of $2 \mathrm{~mm}$ path length was employed. After completing each set of TA experiments, the absorption spectra of all samples were carefully checked to rule out the presence of artifacts or spurious signals arising from, for example, degradation or photo-oxidation of the samples in question.

\section{Nanosecond transient absorption measurements}

The nanosecond transient absorption spectra were obtained using nanosecond flash photolysis techniques. Specifically, a tunable excitation pulse was generated using an Optical Parametric Oscillator system (Continuum, Surelite OPO), which was pumped by $355 \mathrm{~nm}$ from the third-harmonic output of a Q-switched Nd:YAG laser (Continuum, Surelite II-10). The time duration of the excitation pulse was ca. $6 \mathrm{~ns}$, and the 11 pulse energy was ca. $2 \mathrm{~mJ} /$ pulse. A CW Xe lamp $(150 \mathrm{~W})$ was used as the probe light source for the transient absorption measurement. The probe light was collimated on the sample cell and was spectrally resolved using a $15 \mathrm{~cm}$ monochromator (Acton Research, SP150) equipped with a 600 grooves $/ \mathrm{mm}$ grating after passing the sample. The spectral resolution was approximately $3 \mathrm{~nm}$ for the transient absorption experiment. The light signal was detected using an avalanche photodiode (APD). The output signal from the APD was recorded using a $500 \mathrm{MHz}$ digital storage oscilloscope (Lecroy, WaveRunner 6050A) for the temporal profile measurement. Since the triplet-state dynamics of molecules in solution are strongly dependent on the concentration of oxygen molecules dissolved in solution, we attempted to remove oxygen by degassing with Ar gas for 1 hour.

\section{Sub-nanosecond transient absorption measurements}


The sub-nanosecond time-resolved transient absorption spectrometer consists of Optical Parametric Amplifiers (Palitra, Quantronix) pumped by a Ti:sapphire regenerative amplifier system (Integra-C, Quantronix) operating at $1 \mathrm{kHz}$ repetition rate for excitation pulse, Ti:sapphire laser (Maitai BB, SpectraPhysics) which provides a repetition rate of $80 \mathrm{MHz}$ with $\sim 100 \mathrm{fs}$ pulses and an optical detection system. The generated OPA pulses, which were used as pump pulses, had a pulse width of $\sim 100 \mathrm{fs}$ and an average power of $100 \mathrm{~mW}$ in the range 280-2700 nm. $80 \mathrm{MHz}$ pulse train in the range from 710-990 nm from Ti:sapphire laser generates supercontinuum pulses, which were used as probe pulses, by photonic crystal fiber (FemtoWHITE 800, NKT Photonics). To obtain the time-resolved transient absorption difference signal $(\Delta \mathrm{A})$ at a specific time, randomly-interleaved-pulse-train method $^{7}$ is used with photodiodes (FPD510-V, Menlosystems) and oscilloscope.

\section{Computational details are described below.}




\section{Steady state absorption \& emission data}

Table S1 Summary of SF rate, quantum yield, fluorescence lifetime and other parameters

\begin{tabular}{|c|c|c|c|c|c|c|c|c|}
\hline Solvents & Samples & $\tau_{\mathrm{MEG} 1}{ }^{\mathrm{a}}$ & $\mathrm{QY}_{\mathrm{Fl}}{ }^{\mathrm{b}}$ & $\tau_{\mathrm{F} \mid}(n s)^{c}$ & $\mathrm{~K}_{\mathrm{r}} / 10^{7}$ & $\mathrm{~K}_{\mathrm{nr}} / 10^{7}$ & $\mu_{e m, i}{ }^{d}$ & $\mu_{e m, f^{d}}$ \\
\hline Tol & \multirow{3}{*}{ Bis-PBI 2} & 370 ps & 0.67 & 5.4 & 12.4 & 6.10 & 6.87 & 5.28 \\
\hline TCE & & $42 \mathrm{ps}$ & 0.35 & 7.3 & 4.77 & 8.86 & 5.41 & 3.48 \\
\hline $\mathrm{BCN}$ & & 22 ps & 0.18 & 7.7 & 2.32 & 10.6 & 4.48 & 2.38 \\
\hline Tol & \multirow{3}{*}{ Bis-PBI 3} & - & 0.85 & 5.7 & 14.9 & 2.6 & 5.29 & 5.41 \\
\hline TCE & & $55 \mathrm{ps}$ & 0.45 & 6.2 & 7.24 & 8.84 & 5.67 & 3.91 \\
\hline $\mathrm{BCN}$ & & $26 \mathrm{ps}$ & 0.06 & 3.3 & 1.78 & 27.9 & 5.96 & 2.05 \\
\hline
\end{tabular}

a. Measured by TA

b. Relative quantum yield is determined by Rhodamine $6 \mathrm{G}$ in EtOH.

c. Fl. lifetime is averaged over $560-730 \mathrm{~nm}$.

d. Detailed in FLUPS parts

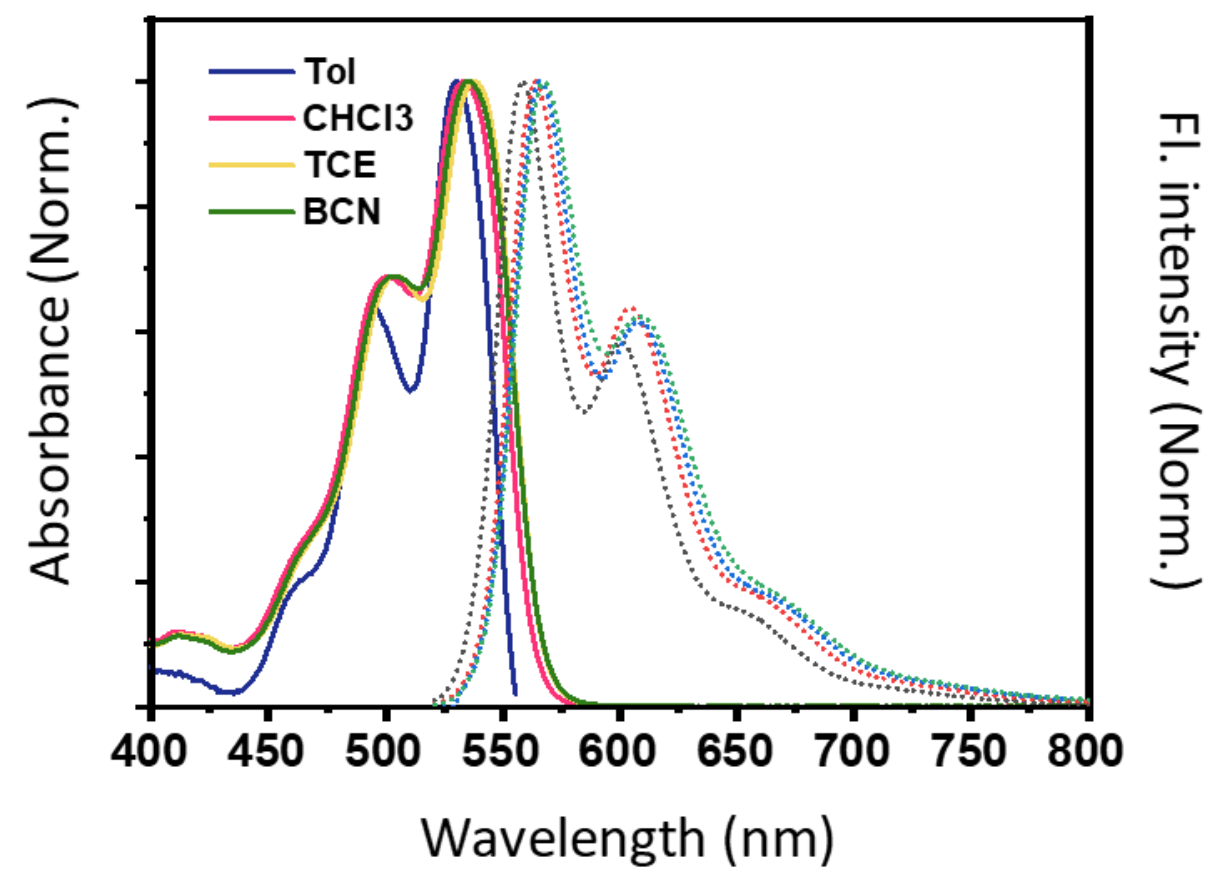

Figure S1. Steady state absorption and fluorescence spectra of ref-Monomer. The quantum yields of ref-Monomers are close to 1 

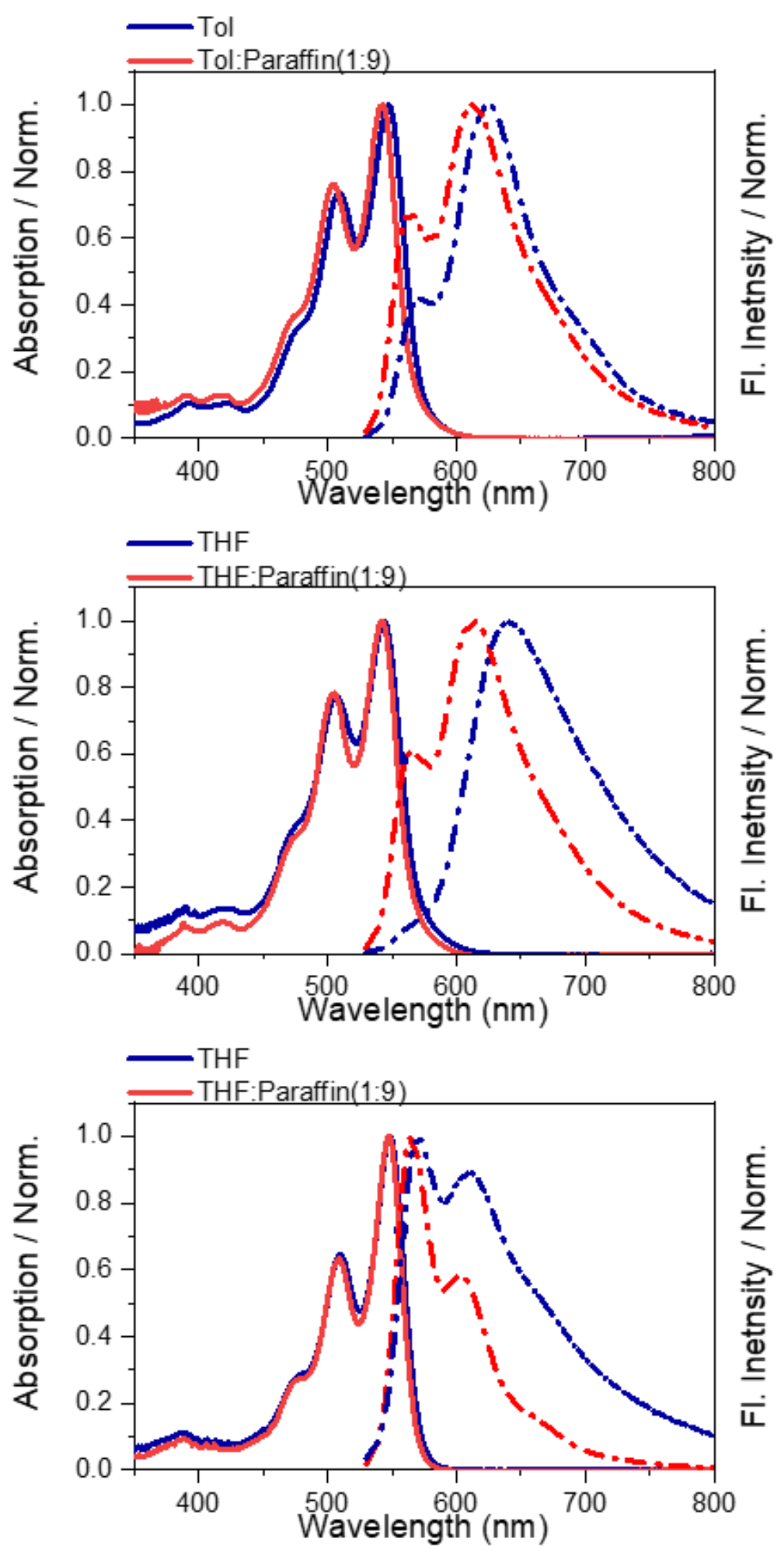

Figure S2. Steady state absorption and fluorescence spectra of Bis-PBI 2 (top and middle) and Bis-PBI 3 (bottom) in viscous medium. 
a Toluene

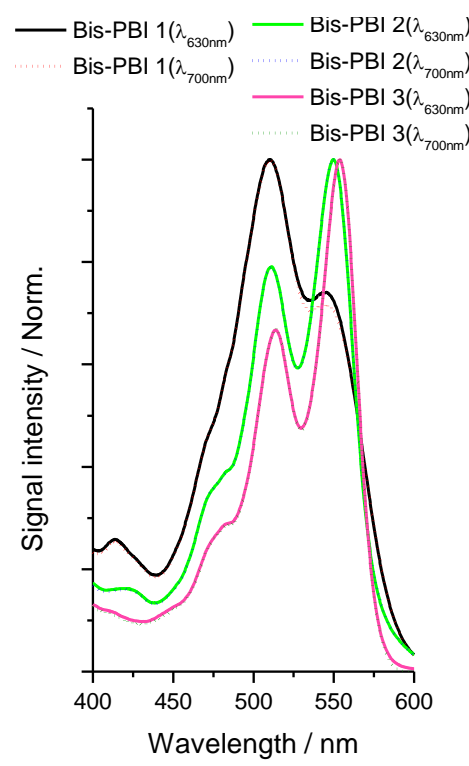

b TCE

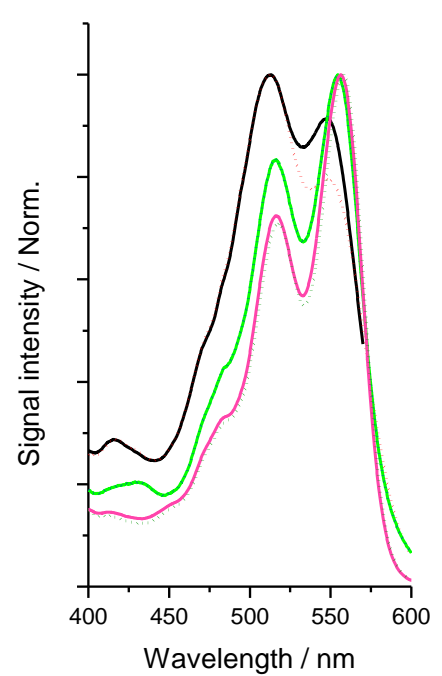

c $\mathrm{BCN}$

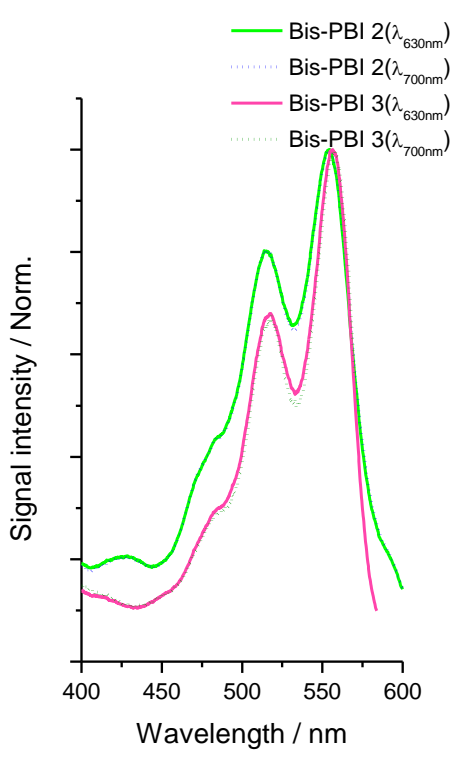

Figure S3. Steady state excitation spectra of Bis-PBI 1, Bis-PBI 2 and Bis-PBI 3 in viscous medium. Compared to Bis-PBI 1, the excitation spectra of Bis-PBI 2 and Bis-PBI 3 are insensitive to the detection wavelength. 


\section{3. fs-Transient absorption data}

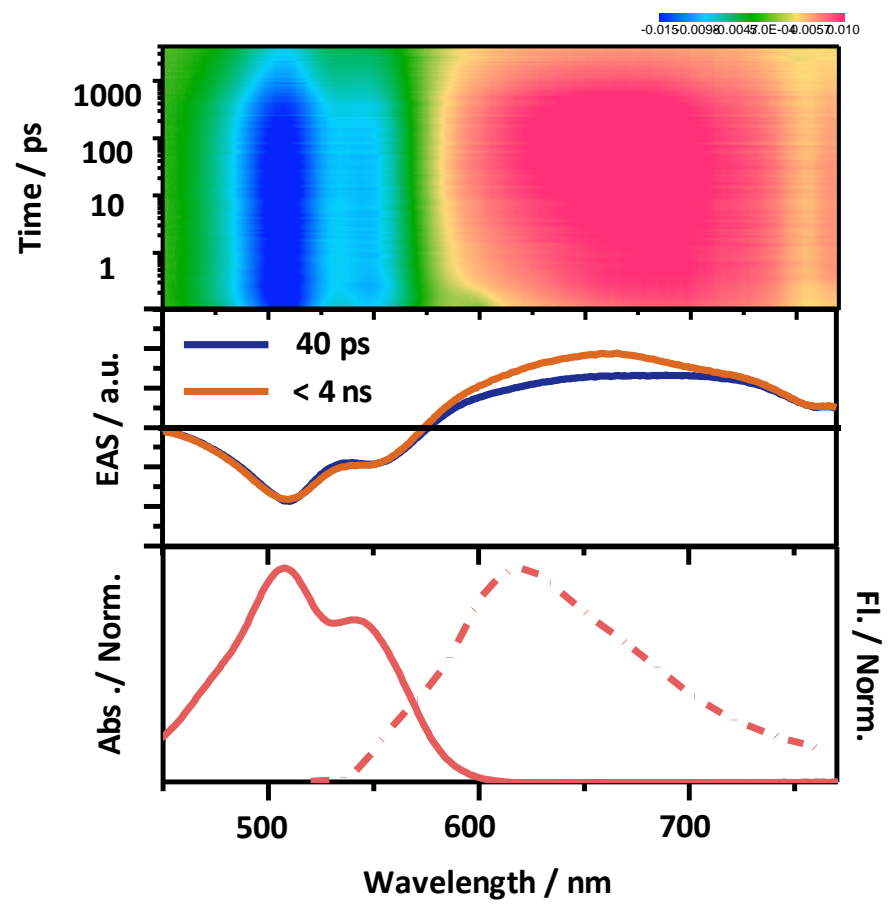

Figure S4. TA results of Bis-PBI 1 in toluene (Top: 2D contour map, middle: EAS1 (40ps) and EAS2 (long time), and bottom: steady state absorption and fluorescence spectra)

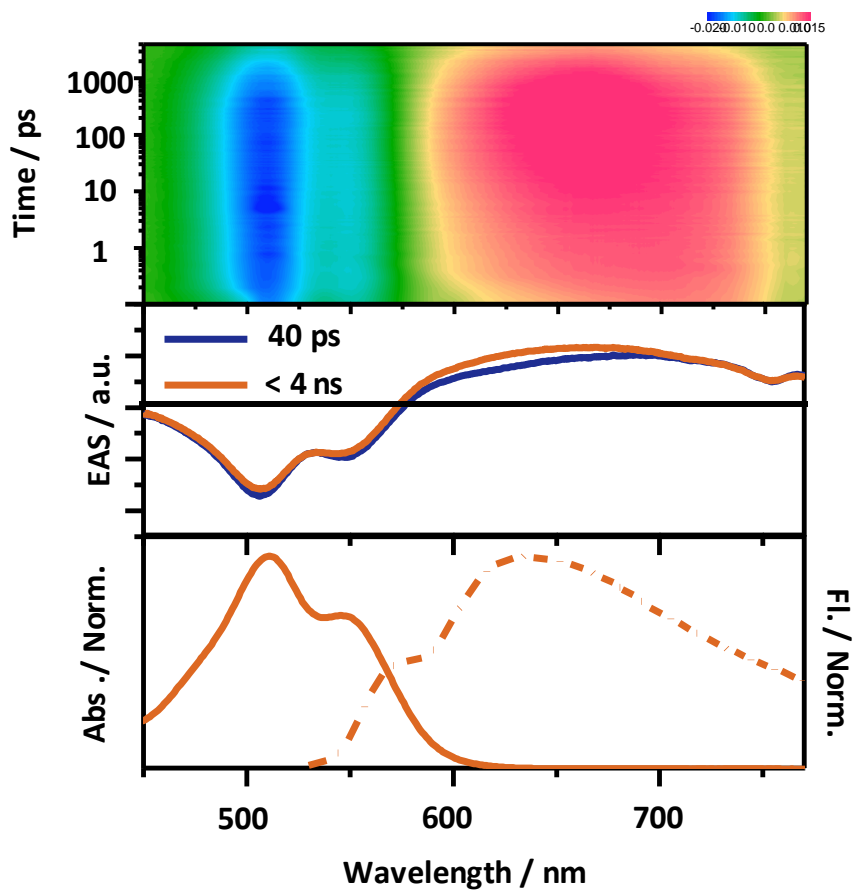

Figure S5. TA spectra (top panel) and EAS (middle panel) of Bis-PBI 1 in TCE. (Top: 2D contour map, middle: EAS1(40ps) and EAS2 (long time), and bottom: steady state absorption and fluorescence spectra) 


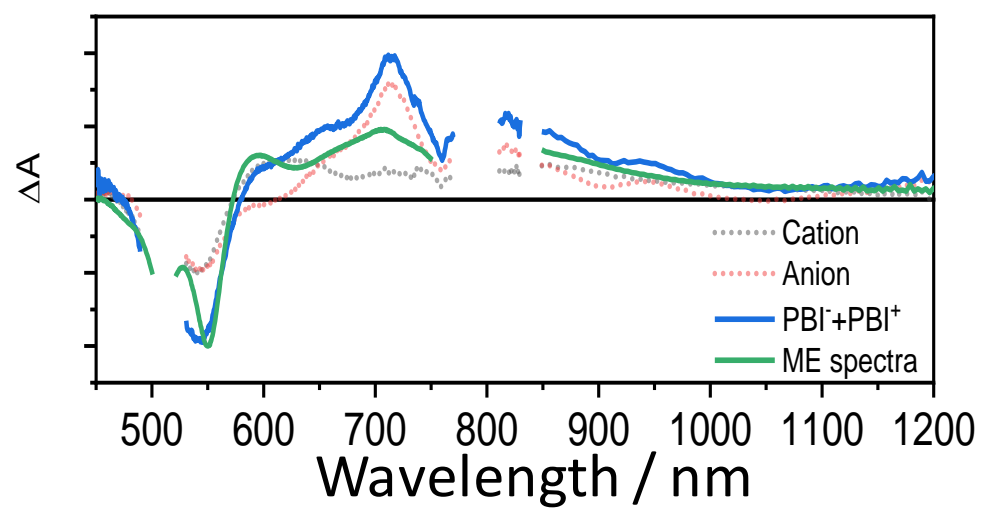

Figure S6 Simulation of transient absorption spectra (blue solid line) of a fully separated ion pair $\left(\mathrm{PBI}^{+} / \mathrm{PBI}^{-}\right)$state. $\left(\right.$for $\mathrm{PBI}^{+}$: ref-PBI + Tetracyanoethylene $0.3 \mathrm{M}$ in $\mathrm{BCN}$ and for $\mathrm{PBI}^{-}: \mathrm{PBI}+$ Dimethylaniline $0.3 \mathrm{M}$ in $\mathrm{BCN}$ ) after photoexcitation at 510 nm. ${ }^{16}$ 

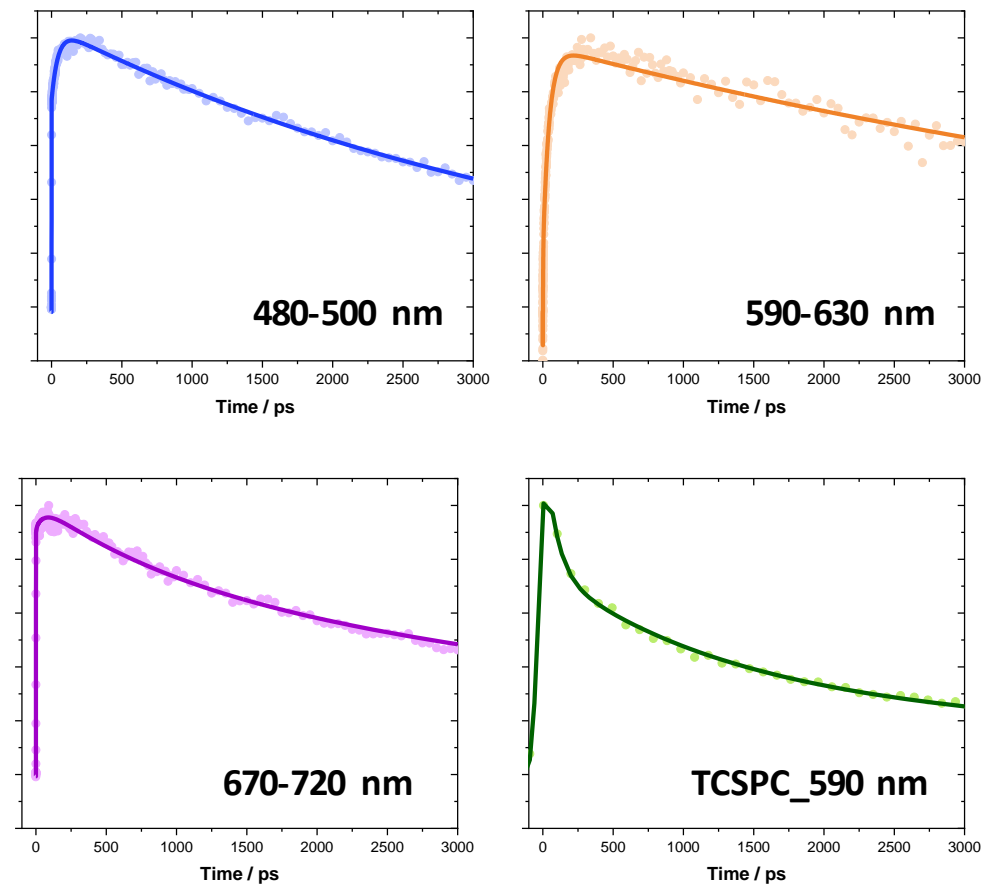

Figure S7. Band integrals for TA kinetics of Bis-PBI 2 in TCE.

Time constants: $\tau_{1}: 3.8-5 \mathrm{ps}, \tau_{2}: 47.6-60 \mathrm{ps}, \tau_{3}: 900 \mathrm{ps}, \tau_{4}: 3800-7000 \mathrm{ps}$
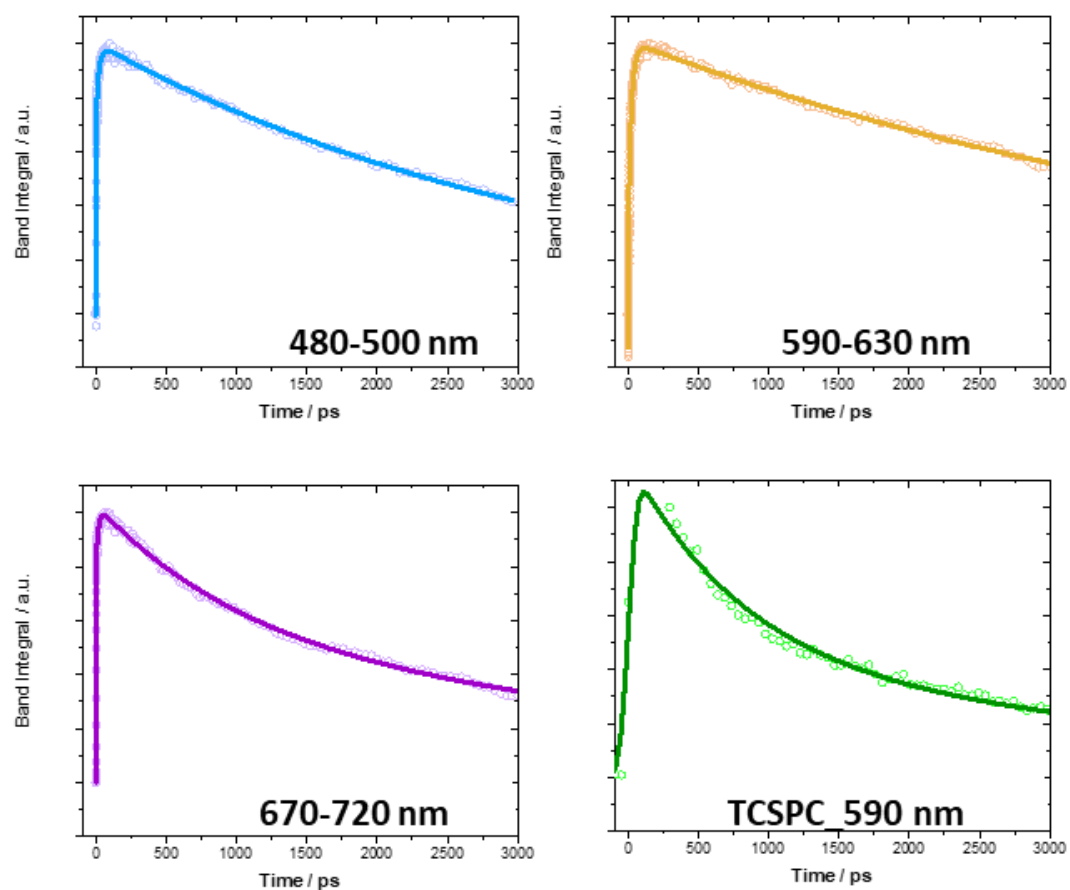

Figure S8. Band integrals for TA kinetics of Bis-PBI 2 in BCN. Time constants: $\tau_{1}: 0.4-4.7 \mathrm{ps}, \tau_{2}: 17.3-23.8 \mathrm{ps}, \tau_{3}: 1039 \mathrm{ps}, \tau_{4}: 3400-5049 \mathrm{ps}$ 

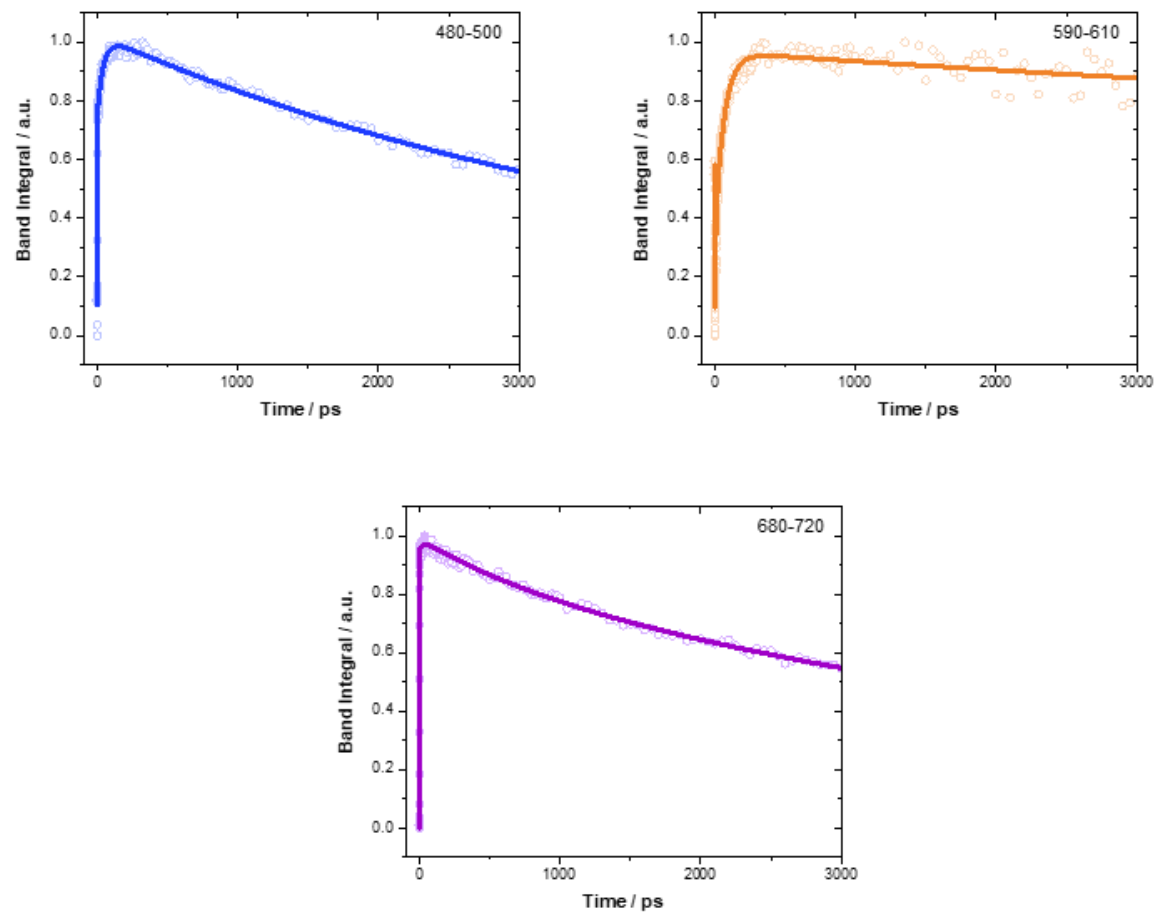

Figure S9. Band integrals for TA kinetics of Bis-PBI 3 in TCE.

Time constants: $\tau_{1}: 0.4-2$ ps, $\tau_{2}: 17-43$ ps, $\tau_{3}: 790-1038$ ps, $\tau_{4}: 4267-6553$ ps
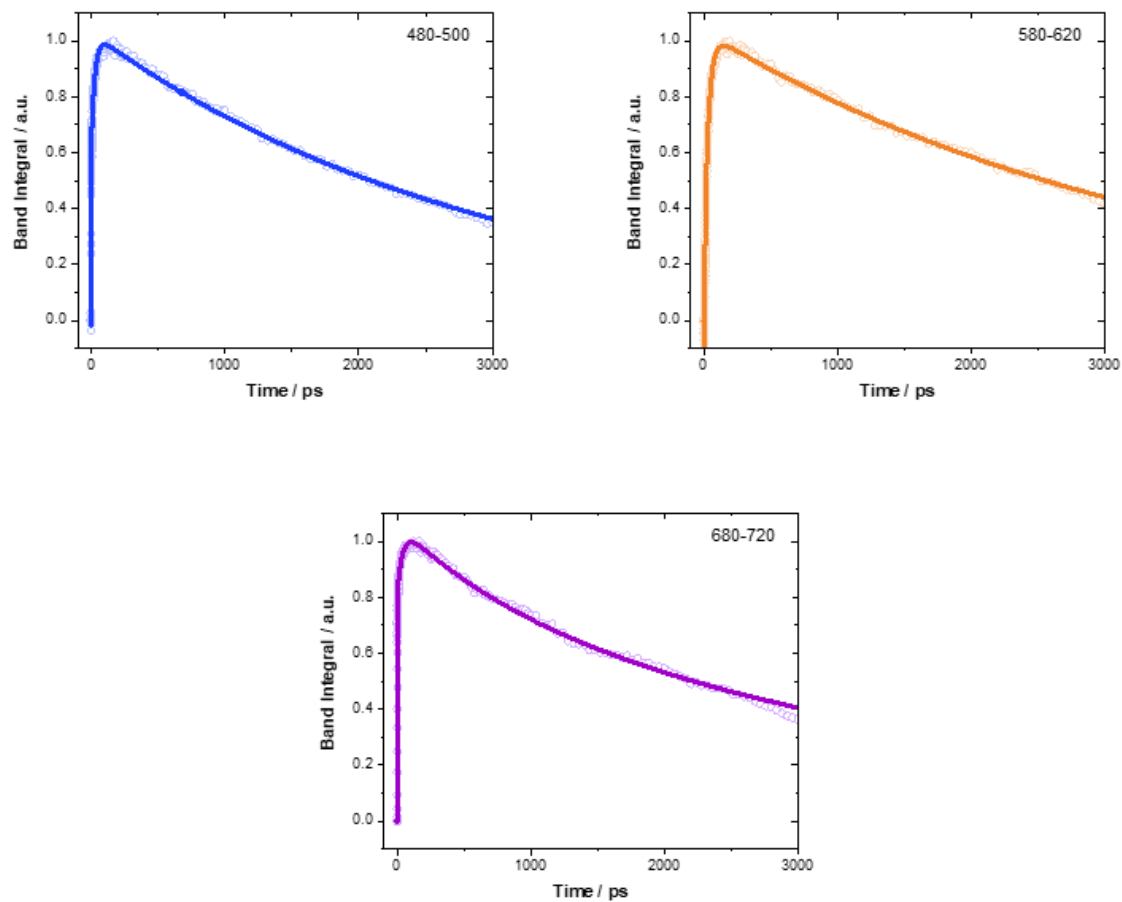

Figure S10. Band integrals for TA kinetics of Bis-PBI 3 in BCN.

Time constants: $\tau_{1}: 1.73-4.7$ ps, $\tau_{2}: 26.5-39.5$ ps, $\tau_{3}: 800$ ps, $\tau_{4}: 2960-4000$ ps 

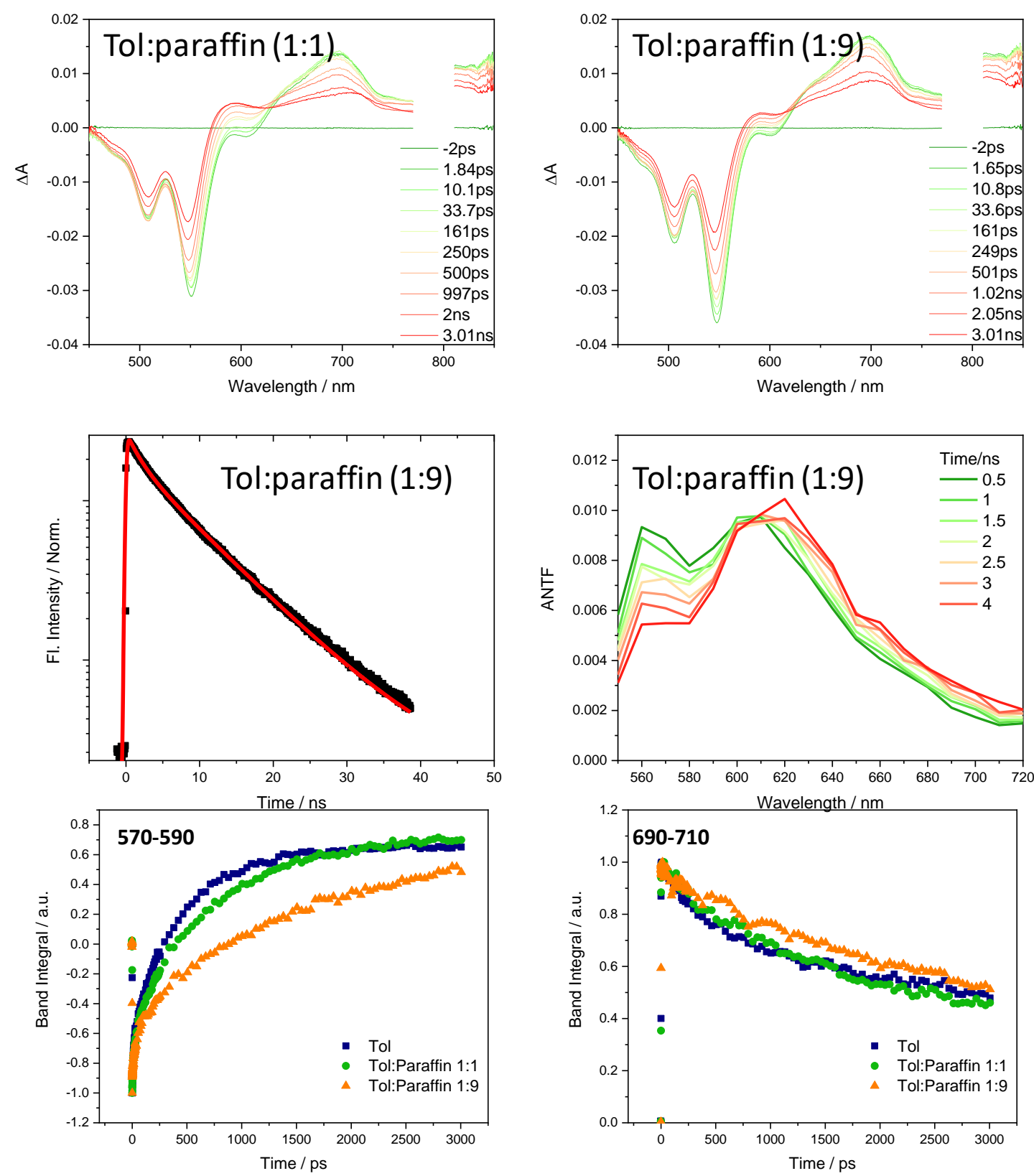

Figure S11. Viscosity controlled TA spectra \& TCSPC measurements of Bis-PBI 2 in tol:Paraffin oil (v:v) and band integrals in TT and LE bands. 


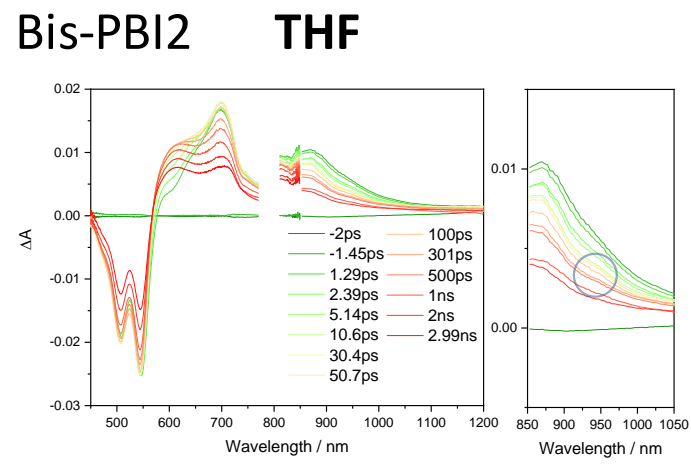

THF:Paraffin (1:1)

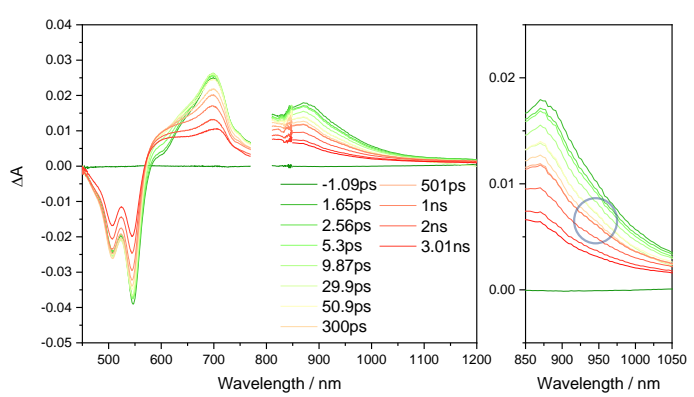

THF:Paraffin (1:9)
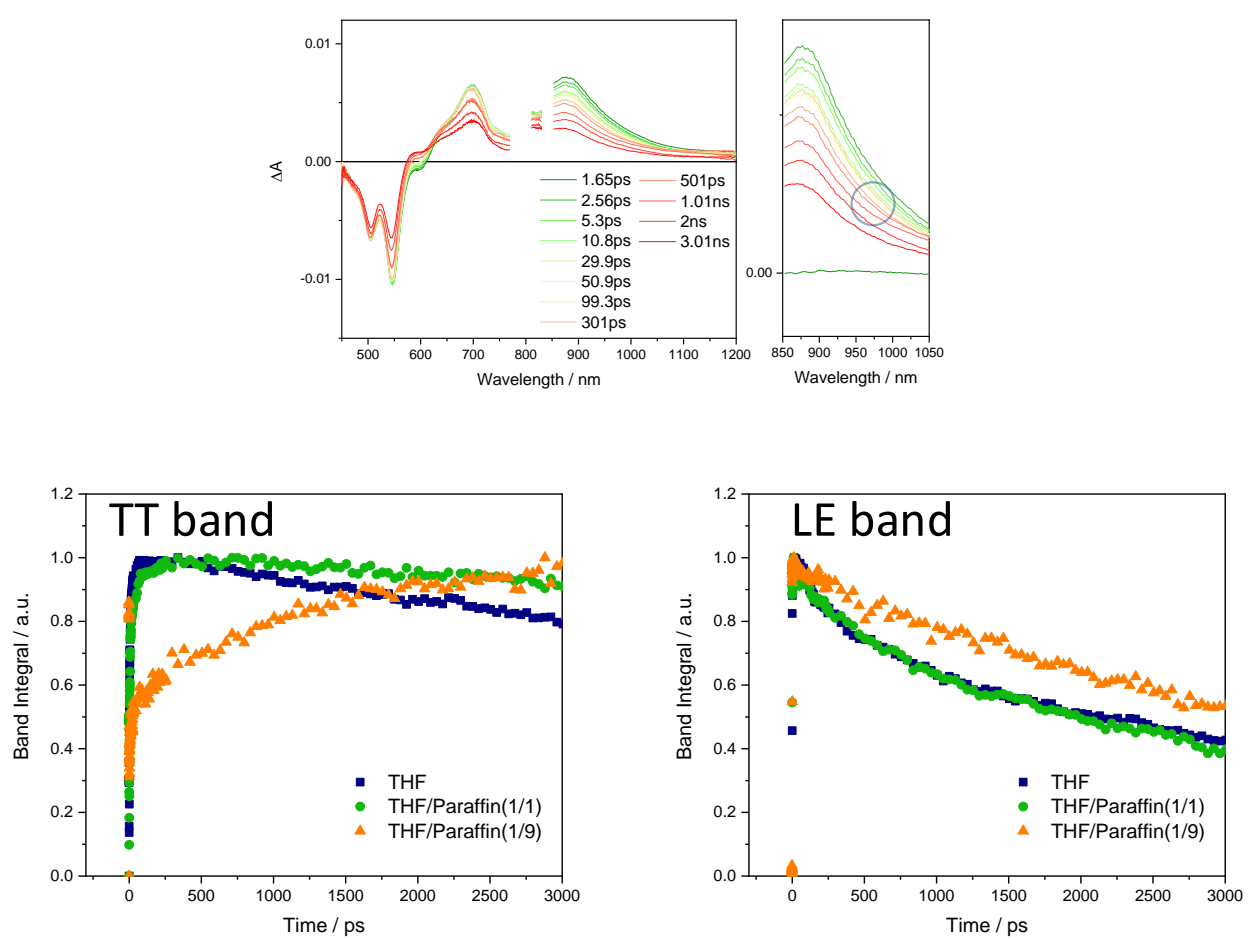

Figure S12. Viscosity controlled TA spectra of Bis-PBI 2 in THF:Paraffin oil (v:v) and band integrals in TT and LE bands. 


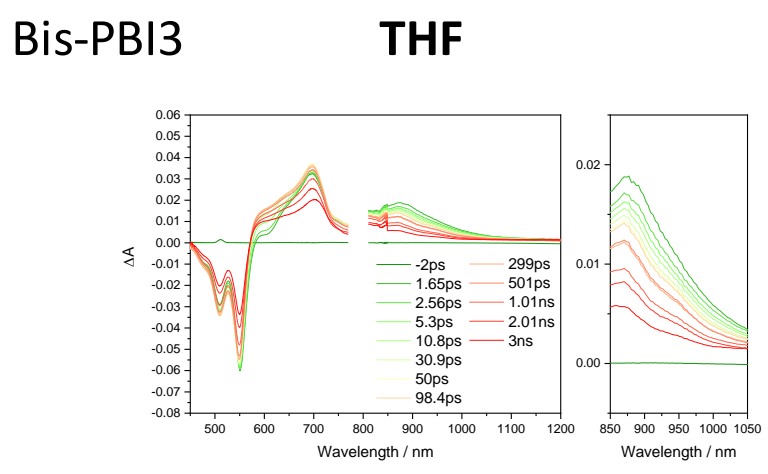

THF:Paraffin (1:1)

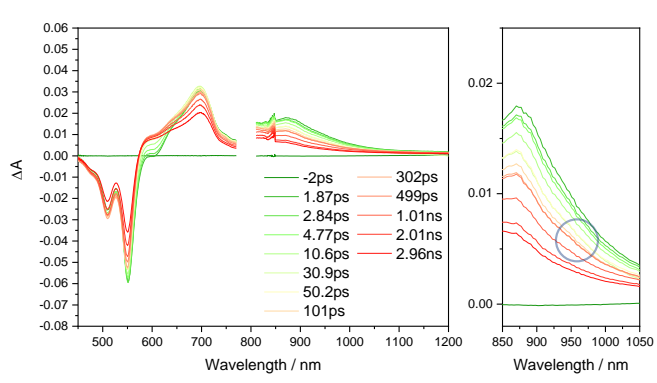

THF:Paraffin (1:9)
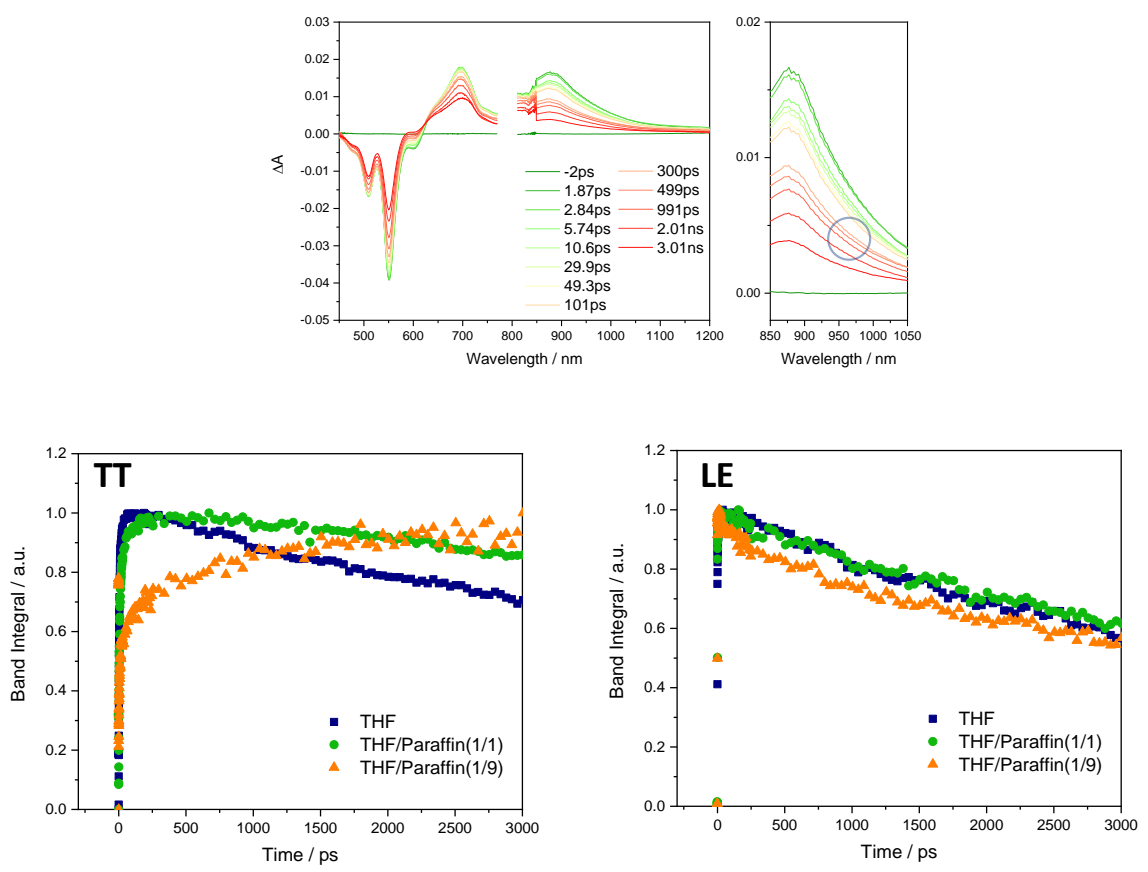

Figure S13. Viscosity controlled TA spectra of Bis-PBI 3 in THF:Paraffin (v:v) and band integrals in TT and LE bands. 


\section{4. ns-Transient absorption data}
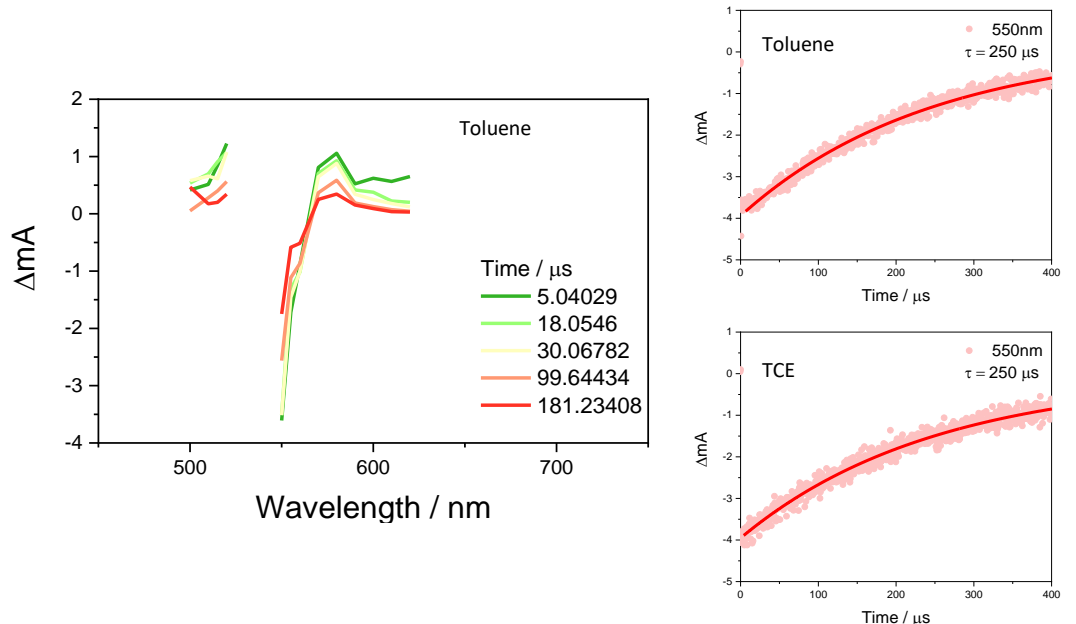
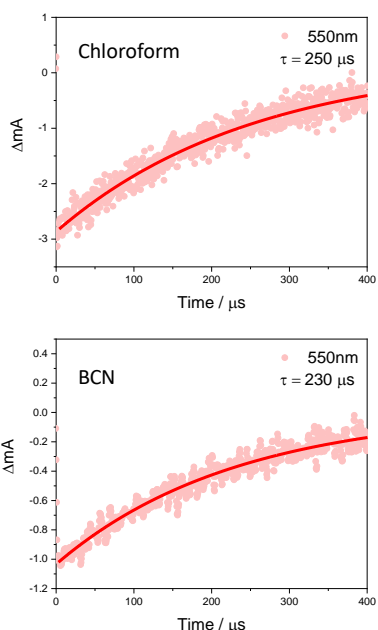

Figure S14. ns-TA spectra of Bis-PBI 2 in toluene (left) and solvent-dependent ns-TA decay profiles (right). (All solvents are saturated by Ar)
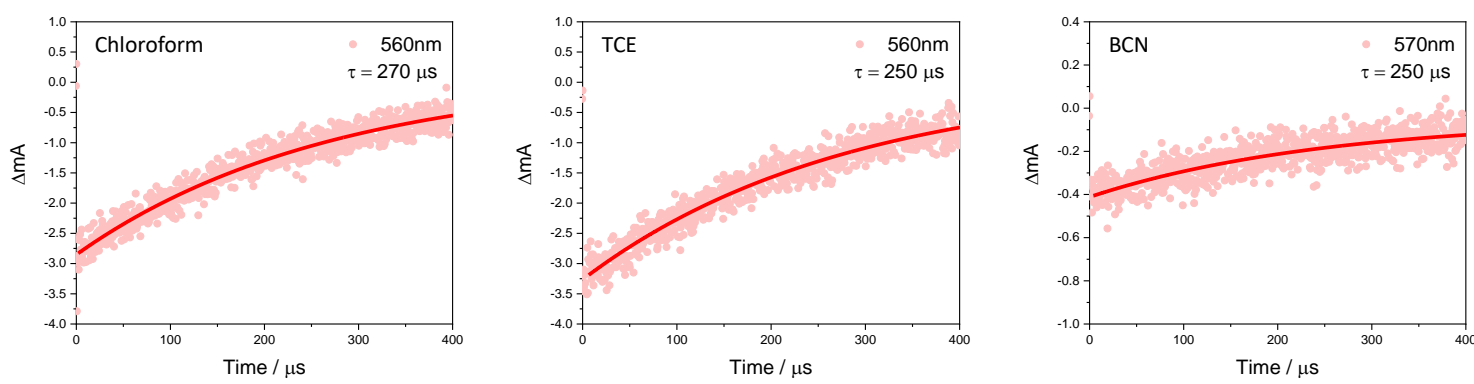

Figure S15. Solvent-dependent ns-TA decay profiles of Bis-PBI 3. (All solvents are saturated by Ar) 


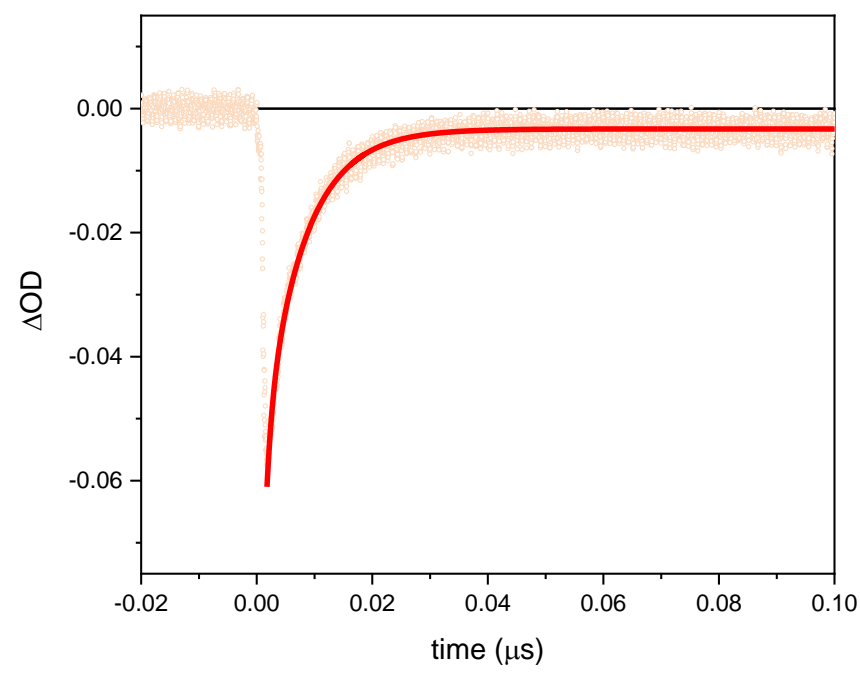

Figure S16. RIPT decay profile of Bis-PBI 2 in BCN $\left(\lambda_{\mathrm{exc}}=520\right.$ and $\left.\lambda_{\mathrm{em}}=560 \mathrm{~nm}\right)$. 


\section{TSCPC data}
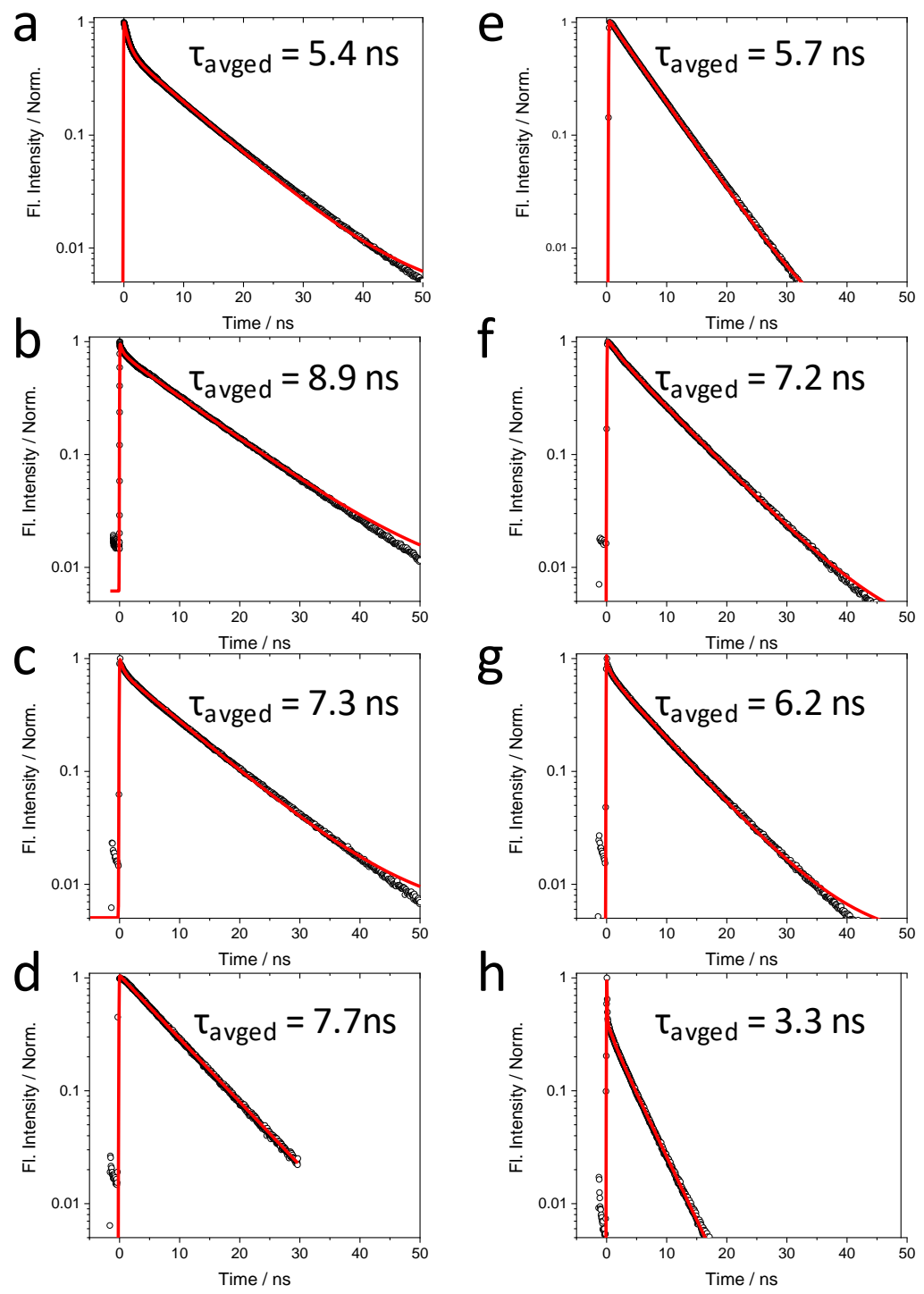

Figure S17. Integrated TCSPC decay (550-720 nm) of Bis-PBI 2 (left) and Bis-PBI 3 (right). (a and e: toluene, b and f: $\mathrm{CHCl}_{3}, \mathrm{c}$ and $\mathrm{g}$ : TCE and $\mathrm{d}$ and $\left.\mathrm{h}: \mathrm{BCN}\right)\left(\lambda_{\mathrm{exc}}=400 \mathrm{~nm}\right)$ 
a

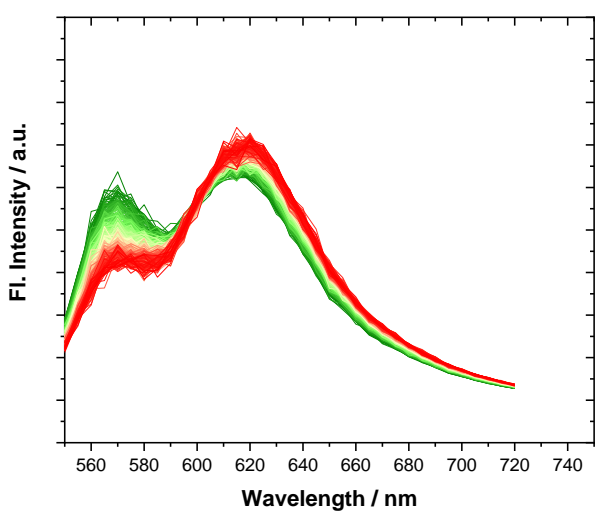

b

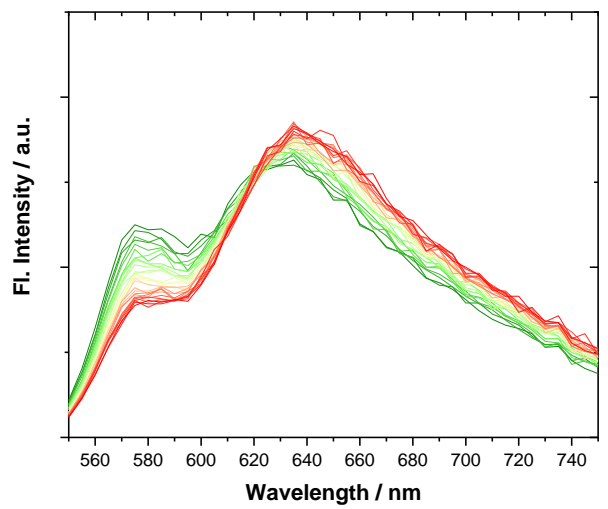

C

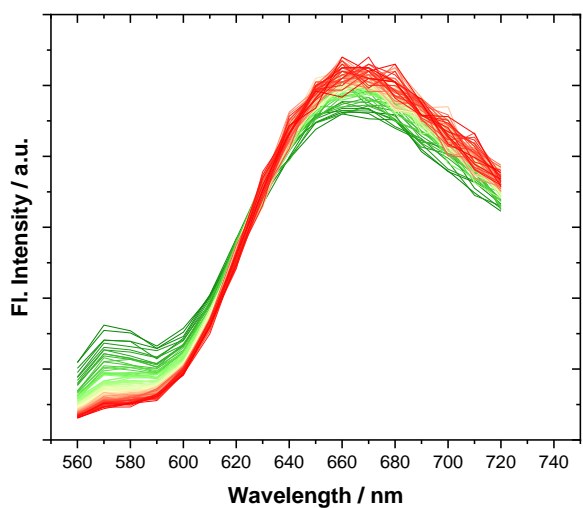

Figure S18. ANTF of Bis-PBI 2 from 0.5 to 3 ns using TCSPC (from green to red). (a: toluene, b: TCE and c: BCN). All spectra were corrected by the steady state fluorescence spectra. 


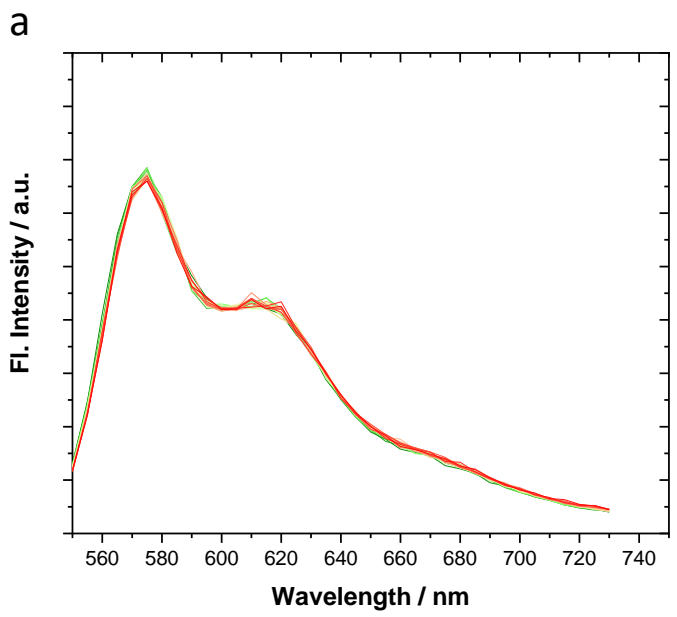

b

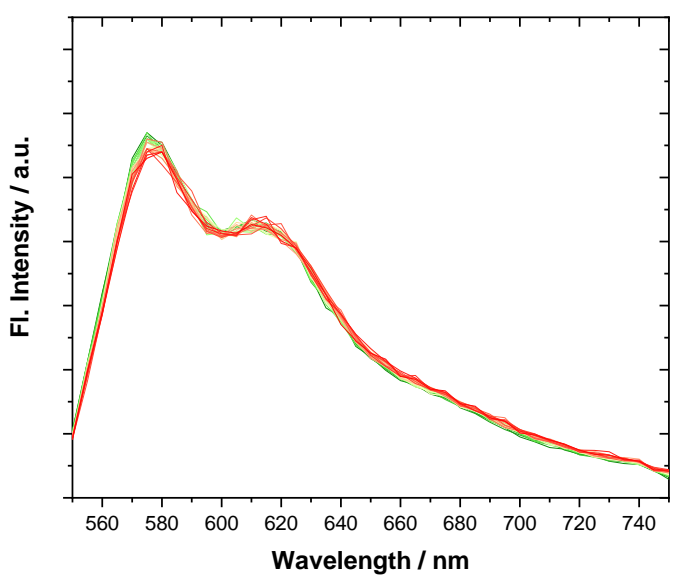

C

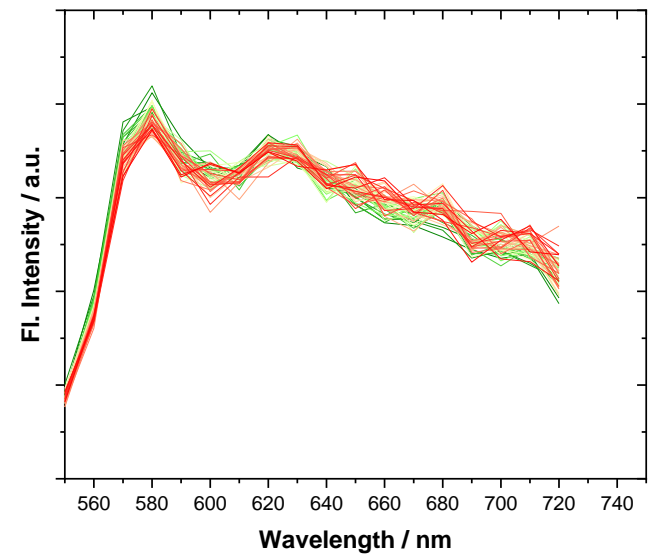

Figure S19. ANTF of Bis-PBI 3 from 0.5 to 3 ns using TCSPC (from green to red). (a: $\mathrm{CHCl}_{3}, \mathrm{~b}$ : TCE and c: $\mathrm{BCN}$ ). All spectra were corrected by the steady state fluorescence spectra. 


\section{FLUPS data}

For Bis-PBI 2, transient fluorescence spectra were corrected by fluorescence spectra from TCSPC measurements at 500 ps. And transient fluorescence spectra of Bis-PBI 3 were corrected by the steady state fluorescence spectra as the spectral evolution with $\sim$ ns time scale is not observed.

To analyze transient fluorescence, we used two methods which are the area normalized transient fluorescence (ANTF) and transient transition dipole moment (TDM).

\section{Area normalized transient fluorescence spectra ${ }^{8}$}

For ANTF, we used the following equation;

$$
I(v, t)=I_{s S}(v) \frac{\sum_{j} \alpha_{j}(v) e^{-t / \tau_{j}(v)}}{\sum_{j} \alpha_{j}(v) \tau_{j}(v)} \quad \cdots
$$

$\mathrm{I}_{\mathrm{ss}}(\mathrm{v})$ : the steady-state fluorescence (or transient spectra at $500 \mathrm{ps}$ ) intensity at $\mathrm{v}$, which is correction factor.

$\alpha_{j}(v)$ and $\tau_{j}(v)$ : the values of the fit parameters

\section{Time-dependent $\mu_{\mathrm{em}}$ calculation method. ${ }^{9}$}

\section{Step 1. Emission dipole moment $\left(\mu_{\mathrm{em}}\right)$}

$$
\mu_{e m}=1.7857 \times 10^{3}\left[\frac{1}{n^{3}} \frac{1}{f(n)} \frac{k_{r a d}}{\tilde{v}_{f}^{3}}\right] \quad \cdots
$$

$\mathrm{k}_{\mathrm{rad}}$ : the radiative rate constant $\left(\mathrm{s}^{-1}\right) . \mathrm{k}_{\mathrm{rad}}=\Phi_{\mathrm{F}} / \tau_{\mathrm{F}}\left(\Phi_{\mathrm{F}}\right.$ : fluorescence quantum yield and $\tau_{\mathrm{F}}$ : fluorescence lifetime, which is determined by TCSPC measurements.) As to calculate $\mathrm{k}_{\mathrm{rad}}$, we used the averaged fluorescence lifetime. And the fluorescence quantum yields are determined by using reference dye R6G in EtOH.

$\tilde{v}_{f}^{3}:$ the cube of the emission frequency, defined by $\tilde{v}_{f}^{3}=\frac{\int F(v) v^{3} d v}{\int F(v) d v}$ $f(n)=9 n^{2} /\left(2 n^{2}+1\right)^{2}$ : an effective cavity factor ( $n$ is the refractive index of the solvent.)

\section{Step 2. Analysis of the FLUPS spectra}

Using E. Vauthey's method, the transient transition dipole moment was determined by following procedure.

1) Divide the time-resolved fluorescence spectra by $\mathrm{e}^{-(\mathrm{t} / \tau \mathrm{F})}$ to correct for the population decay ${ }^{*}(\tau \mathrm{F}$; fluorescence lifetime) *: As to Bis-PBI 2 in toluene, the fluorescence decay from TCSPC includes the MEG process. To exclude population decay, we used the averaged fluorescence lifetime. The multiexponential decay plot was also used, but its effect is negligible. As the population decay is not fully removed, time-dependent TDM of Bis-PBI 2 in toluene could be underestimated. $F_{\tau}(t)=F(t) / e^{-\left(\frac{t}{\tau_{F}}\right)}$, where $F(\mathrm{t})=F(\breve{v}) /\left(\tilde{v}^{3}\right)$ in the transition dipole moment representation ${ }^{\text {ref }}$ and $\tilde{v}$ is the wavenumver $\left(\mathrm{cm}^{-1}\right)$.

2) Next, the time-dependent TDM traces can be obtained by following equation.

$$
\mu_{e m}=\mu_{e m}(\text { steady state }) \cdot \sqrt{\int_{v 1}^{v 2} F_{\tau}(t) / \int_{v 1}^{v 2} F_{\tau}(t=\infty)} \cdots(4)
$$

$F_{\tau}(t=\infty)$ is a transient fluorescence spectrum after all relaxation processes are finished, which serves as a normalization factor. We used the transient fluorescence spectrum at $300 \mathrm{ps}$ for $F_{\tau}(t=\infty)$. As to slow MEG process of Bis-PBI 2, we apply a same procedure as our mechanical delay of FLUPS is limited. Therefore, the initial TDM of Bis-PBI 2 in toluene is underestimated. However, we can still 
claim that the initial values of TDM decrease with the solvent polarity and the LE character in nonpolar solvent toluene is dominant. 

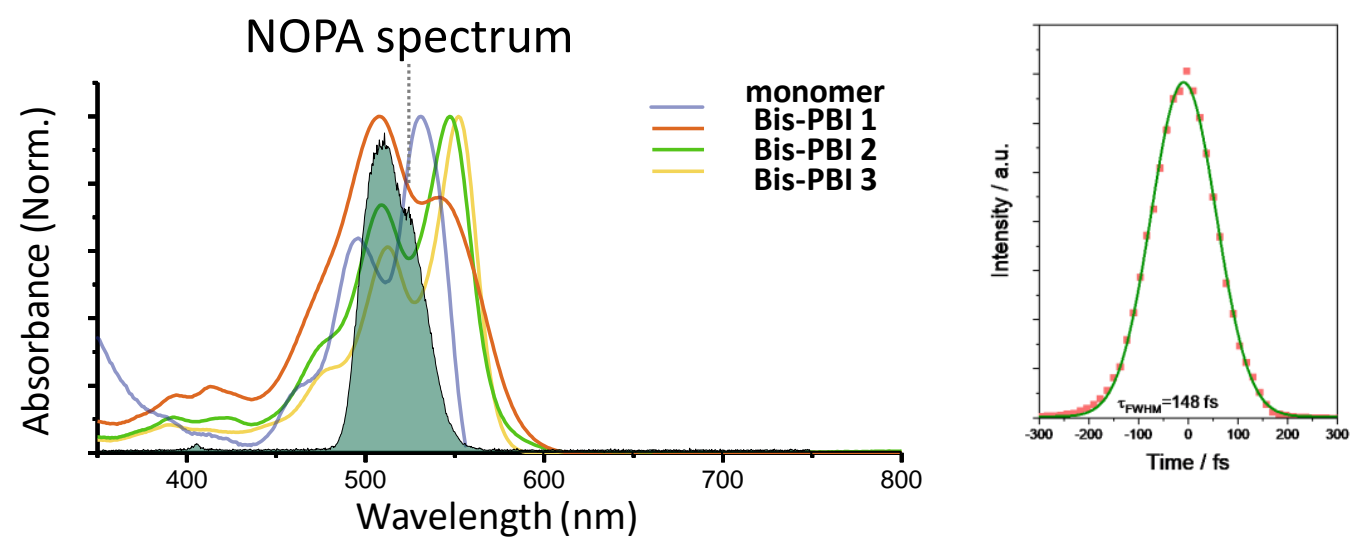

Figure S20. left: Steady state absorption spectra and NOPA spectrum of the pump pulse for the FLUPS measurements and right: Cross-correlation trace between pump and gate at $1325 \mathrm{~nm}$ pulses. 

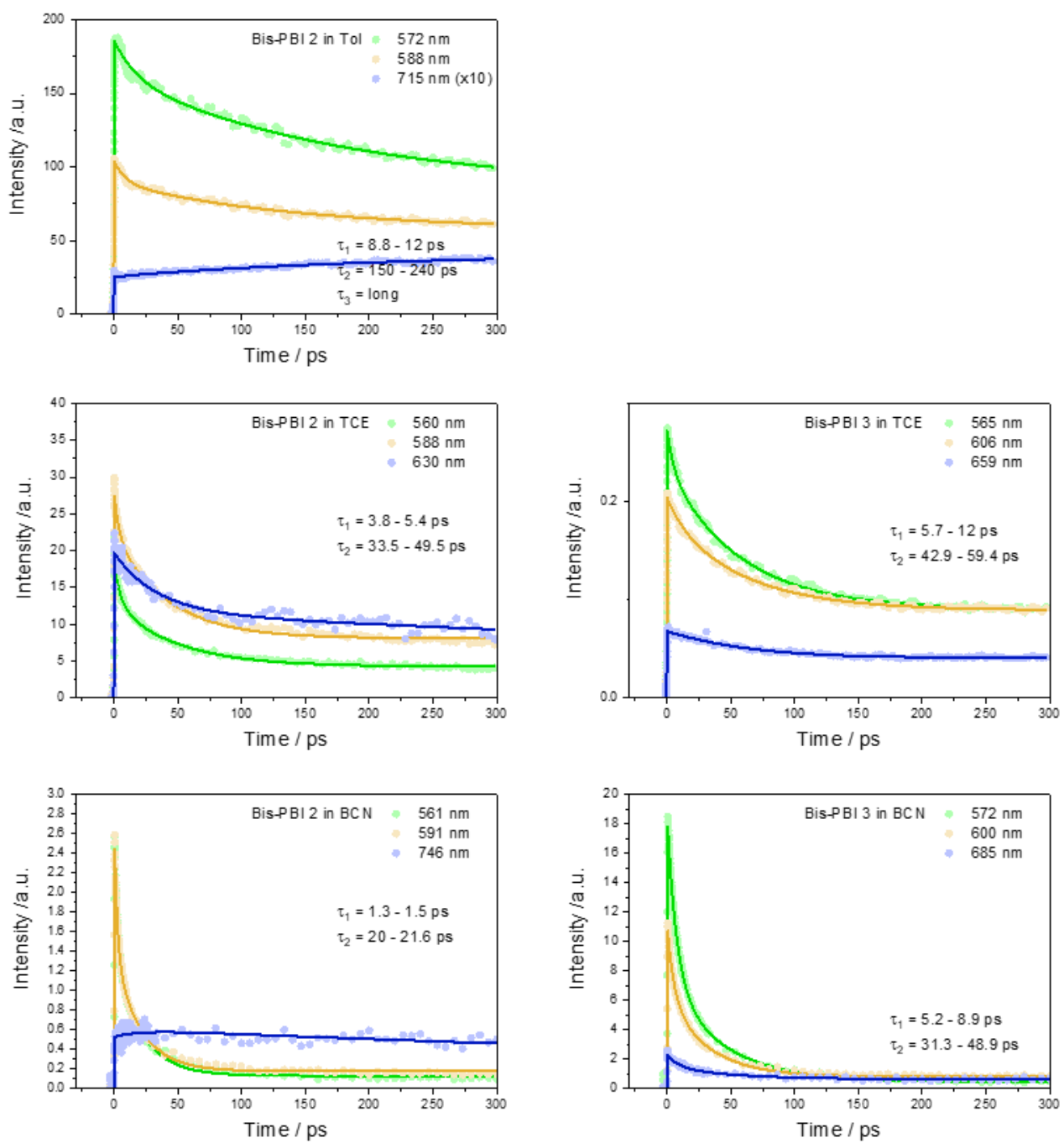

Figure S21. Time resolved fluorescence decay profiles of Bis-PBI 2 (left) and 3 (right). 
Bis-PBI 2
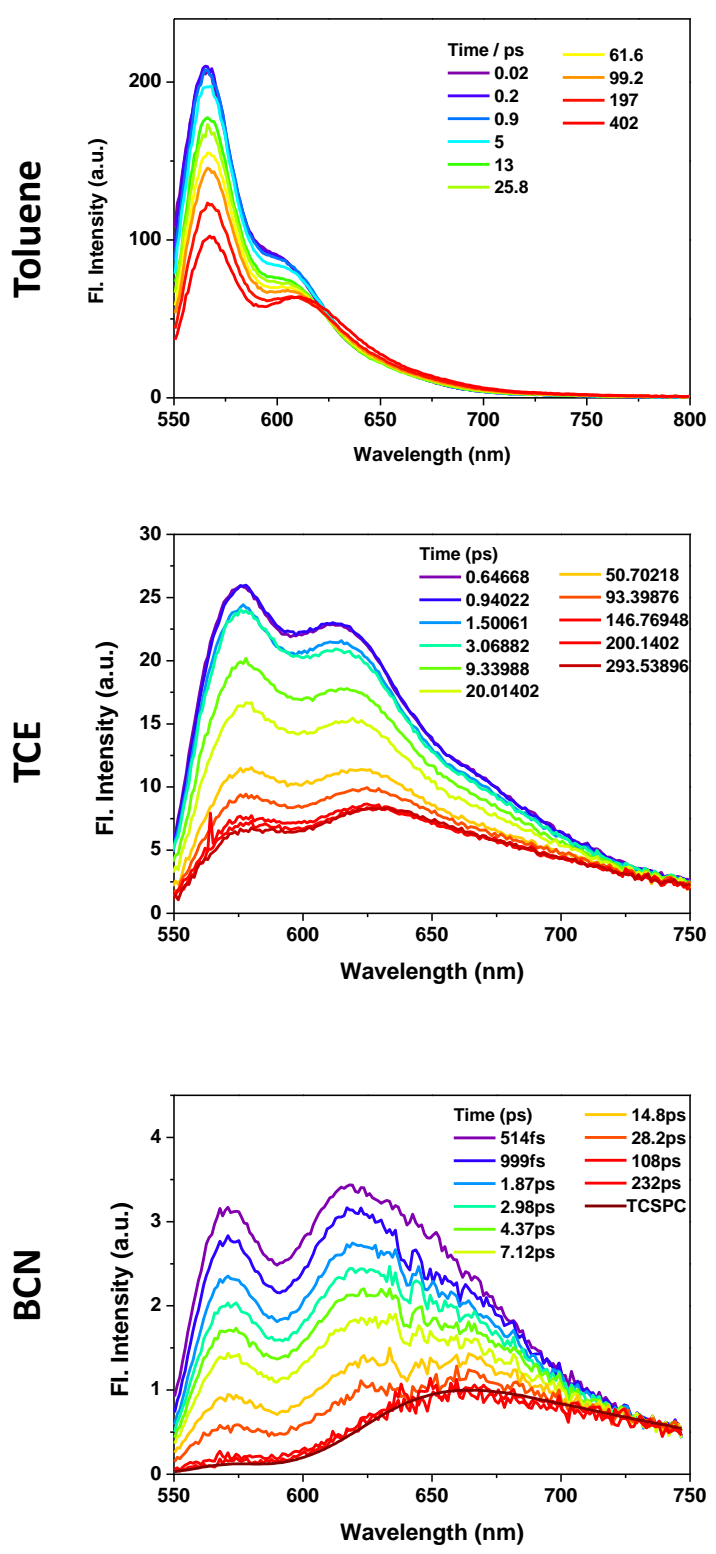

Bis-PBI 3
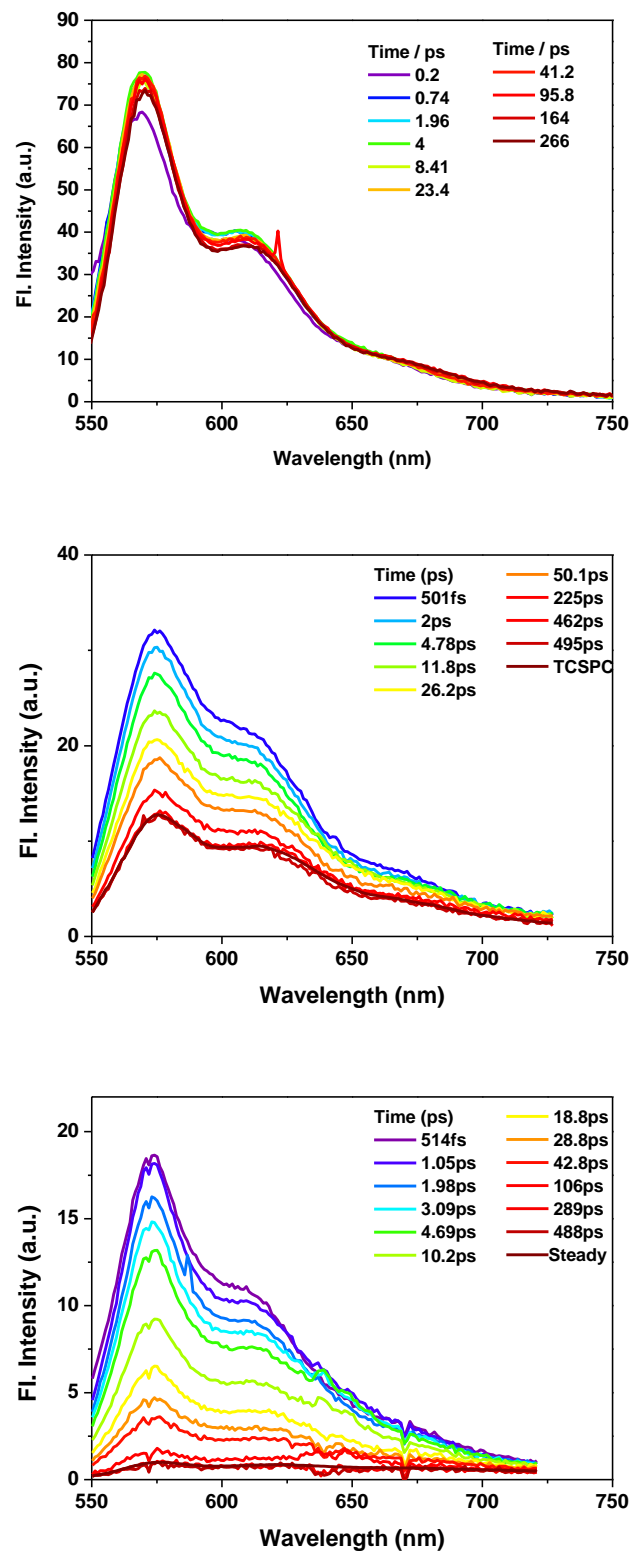

Figure S22. Time resolved fluorescence spectra of Bis-PBI 2 and Bis-PBI 3 

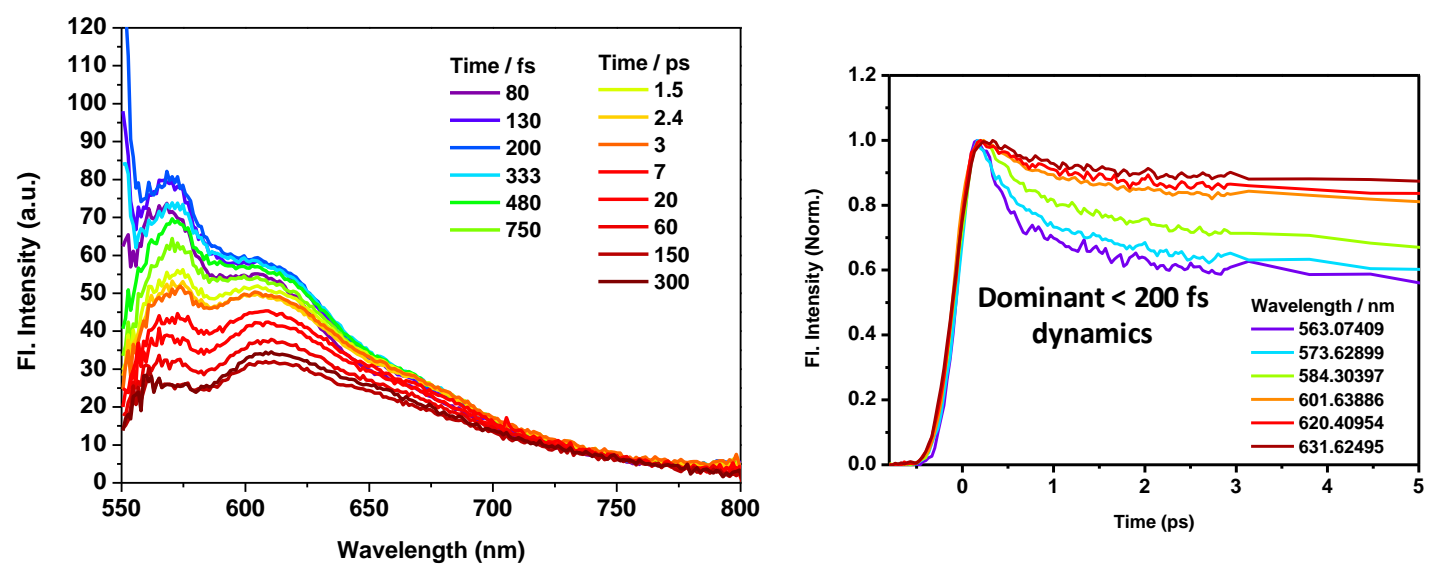

Figure S23. Time resolved fluorescence spectra and decay profiles of Bis-PBI 1 in toluene
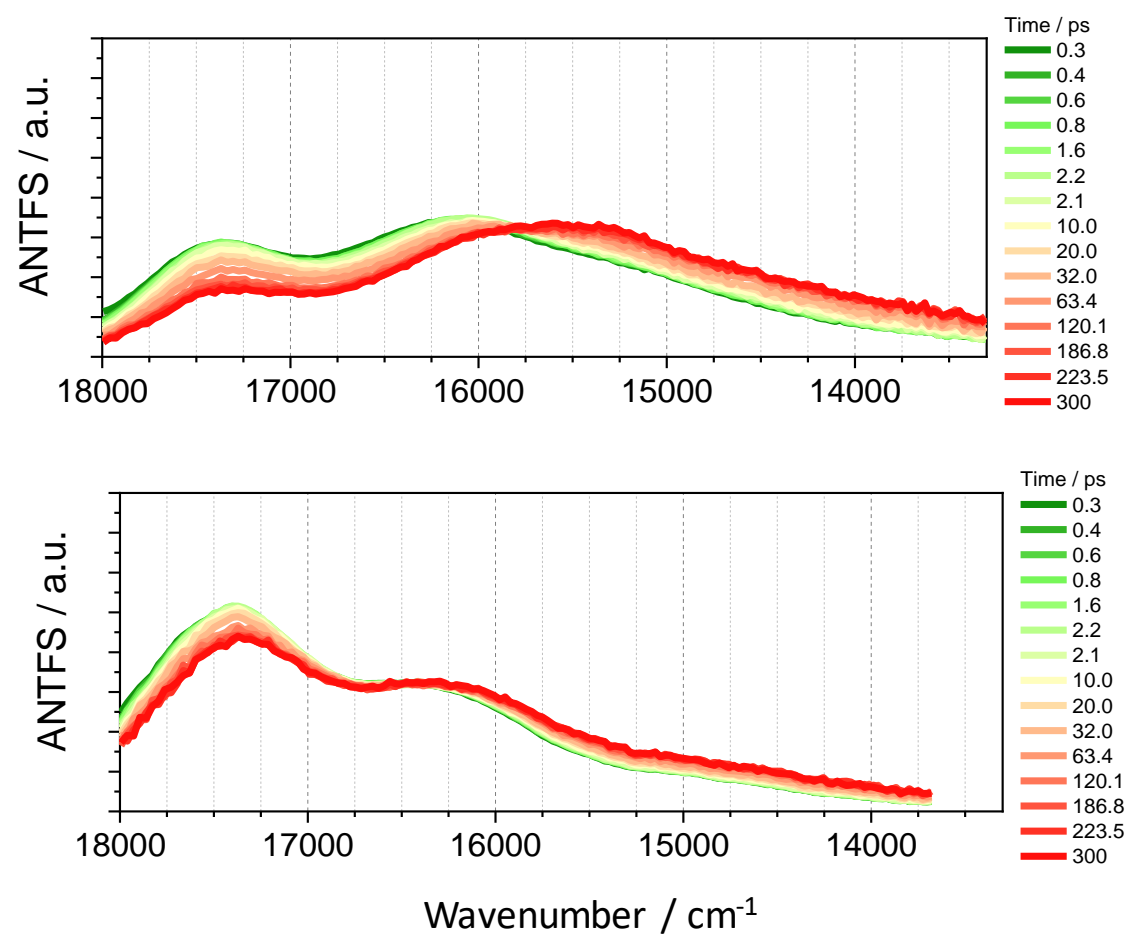

Figure S24. ANTFS of Bis-PBI 2 (top) and 3 (bottom) in TCE. 


\section{Quantum Chemical Simulations}

We performed quantum chemical simulations to describe electronic structures associated with SF. The ground state ( $\left.\mathrm{S}_{0}\right)$ geometries obtained by DFT (B97-D3/def2-SVP) were provided in the previous paper. ${ }^{1}$ The first excited singlet state $\left(\mathrm{S}_{1}\right)$ geometries were optimized with time-dependent DFT (CAM-B3LYP-D/6-31G*). We selected the CAM-B3LYP functional because this functional shows the outstanding performance in describing excited state wavefunction. ${ }^{10}$ Also, CAM-B3LYP could reproduce the excitation energies of PDI monomer and dimer obtained by $\omega$ B97X-D which is the most popular functional to investigate SF energetics. ${ }^{11}$ Dispersion correction was added via Grimme's empirical scheme.

Restricted active-space configuration interaction method with double spin-flip (RAS-2SF) calculations were performed to illustrate the SF energetics and coupling between respective states based on the optimized $\mathrm{S}_{0}$ and $\mathrm{S}_{1}$ geometries. We obtained raw RAS-2SF energies based on the quintet reference state with four electrons in four active orbitals. It is generally accepted that raw RAS-2SF energies are severely overestimated due to the lack of dynamical correlation. This could be improved by adding empirical correction to RAS-2SF raw energies. We have refined RAS-2SF energies to achieve the TDCAM-B3LYP-D/6-31G* quality. Procedure to correct raw RAS-2SF energies is detailed in the previous report. ${ }^{11}$

Three-state model kinetics was suggested by Prof. Krylov, and this simple scheme has been successfully employed to describe SF kinetics. We are interested in the ME formation, and the rate of multiexciton state formation could be approximated as below:

$$
r_{1}=(\mathrm{NAC})^{2} e^{-\alpha \beta \Delta E_{\mathrm{SF}}}=\left(\frac{\|\gamma\|}{\Delta E}\right)^{2} e^{-\alpha \beta \Delta E_{\mathrm{SF}}}
$$

where NAC is nonadiabatic coupling estimated by one-particle transition density matrix and energy difference, $\alpha$ is a parameter in the free-energy relationship and was set to be 0.5 like in the previous reports. ${ }^{12,13} \quad \beta$ is equal to $1 / k_{b} T\left(\mathrm{k}_{\mathrm{b}}\right.$ is the Boltzmann constant and $\mathrm{T}$ is the room temperature of $298 \mathrm{~K}$, and $\Delta E_{\mathrm{SF}}$ represents the energy difference between singlet excited state and multiexciton state.

All quantum chemical simulations were performed with Q-Chem 5.1. ${ }^{14}$

Table S2. Energy correction (in eV) for the CR, ME, and LE contributions (CR: charge resonance, ME: multiexciton, LE: local exciton).

Energy correction obtained for the $\mathrm{S}_{1}$ geometries

\begin{tabular}{cccc}
\hline & Bis-PBI 1 & Bis-PBI 2 & Bis-PBI 3 \\
\hline CR & -1.17 & -1.18 & -1.24 \\
ME & -0.62 & -0.61 & -0.66 \\
LE & -0.90 & -0.47 & -0.47 \\
\hline
\end{tabular}

Table S3. Excitation energies (in eV) and adiabatic wavefunction composition (\%) of SF-relevant states in the first excited singlet state $\left(\mathrm{S}_{1}\right)$ geometry. (GS: ground state, LE: local exciton, $\mathrm{SS}$ : singlet-singlet multiexciton, TT: triplet-triplet multiexciton, ME: multiexciton state (SS+TT), CR: charge resonance, $\mathrm{S}_{\mathrm{n}}$ : the $\mathrm{n}^{\text {th }}$ singlet excited state, Q: quintet state)

Corrected RAS-2SF energies are reported. The excited state is characterized based on the composition with larger than $40 \%$ (The portion is given in bold).

Bis-PBI 2

\begin{tabular}{cccccccc}
\hline$\%$ & Energy & GS & LE & SS & TT & ME & CR \\
\hline S1 (LE) & 2.45 & 0.00 & $\mathbf{7 0 . 1 5}$ & 10.45 & 0.05 & 10.50 & 19.35 \\
\hline
\end{tabular}




\begin{tabular}{cccccccc}
\hline S2 (ME) & 2.50 & 4.20 & 0.36 & 0.27 & $\mathbf{7 7 . 9 2}$ & 78.19 & 21.03 \\
Q & 2.66 & 0.00 & 0.00 & 50.00 & 50.00 & $\mathbf{1 0 0 . 0 0}$ & 0.00 \\
S3 (CR) & 2.93 & 0.63 & 2.58 & 4.43 & 15.97 & 20.41 & $\mathbf{7 6 . 3 8}$ \\
S4 (CR) & 2.96 & 0.04 & 15.85 & 3.32 & 1.49 & 4.81 & $\mathbf{7 9 . 3 0}$ \\
S5 (ME) & 3.16 & 26.37 & 25.96 & 41.55 & 3.06 & $\mathbf{4 4 . 6 2}$ & 3.05 \\
S6 (LE) & 3.19 & 0.00 & $\mathbf{5 9 . 9 2}$ & 37.29 & 0.04 & 37.33 & 2.75 \\
S7 (LE) & 3.56 & 0.00 & $\mathbf{6 1 . 4 6}$ & 35.79 & 0.29 & 36.08 & 2.46 \\
\hline
\end{tabular}

Bis-PBI 3

\begin{tabular}{cccccccc}
\hline$\%$ & Energy & GS & LE & SS & TT & ME & CR \\
\hline $\mathrm{S}_{1}(\mathrm{LE})$ & 2.52 & 0.00 & $\mathbf{6 6 . 8 4}$ & 18.73 & 0.02 & 18.75 & 14.40 \\
$\mathrm{~S}_{2}(\mathrm{ME})$ & 2.59 & 0.16 & 0.18 & 0.23 & $\mathbf{8 1 . 3 7}$ & 81.60 & 18.07 \\
$\mathrm{Q}$ & 2.73 & 0.00 & 0.00 & 50.00 & 50.00 & $\mathbf{1 0 0 . 0 0}$ & 0.00 \\
$\mathrm{~S}_{3}(\mathrm{CR})$ & 2.95 & 0.00 & 0.94 & 2.54 & 16.59 & 19.13 & $\mathbf{7 9 . 9 2}$ \\
$\mathrm{S}_{4}(\mathrm{CR})$ & 2.97 & 0.00 & 10.57 & 5.80 & 0.72 & 6.52 & $\mathbf{8 2 . 9 1}$ \\
$\mathrm{S}_{5}(\mathrm{ME})$ & 3.10 & 0.00 & 34.10 & 62.01 & 0.03 & $\mathbf{6 2 . 0 4}$ & 3.86 \\
$\mathrm{~S}_{6}(\mathrm{ME})$ & 3.13 & 27.24 & 18.93 & 52.94 & 0.47 & $\mathbf{5 3 . 4 1}$ & 0.42 \\
$\mathrm{~S}_{7}(\mathrm{ME})$ & 3.47 & 0.68 & 32.22 & 63.90 & 0.02 & $\mathbf{6 3 . 9 2}$ & 3.18 \\
\hline
\end{tabular}

Table S4. Rate of ME formation in the first excited singlet state $\left(\mathrm{S}_{1}\right)$ geometry estimated by the three-state model.

\begin{tabular}{ccccc}
\hline & State & $|\gamma|$ & $\Delta \mathrm{E}_{\mathrm{SF}}[\mathrm{eV}]$ & $r_{1}$ \\
\hline Bis-PBI 2 & $\mathrm{S}_{1}$ & 0.51 & 0.05 & $3.9 \times 10^{1}$ \\
Bis-PBI 3 & $\mathrm{S}_{1}$ & 0.46 & 0.07 & $1.1 \times 10^{1}$ \\
\hline
\end{tabular}

While the lowest ME states in both Bis-PBI 2 and Bis-PBI 3 show dominant ME character with some CR contribution, high-lying excited singlet states feature stronger mixing between ME and LE. 


\section{Further discussion}

A. SF quantum yield determination

1) GSB method

To calculate singlet fission yield, we first extracted evolution associated spectra corresponding to $\mathrm{S}_{1}$ and ME states (EAS1 and EAS3, respectively). The singlet depletion method (GSB quantization) is not suitable for our system due to the spectral overlap. Next, GSB bands of EAS3 is divided by those of EAS1. To minimize overlap with SE bands, we used GSB bands from 460 to $530 \mathrm{~nm}$.
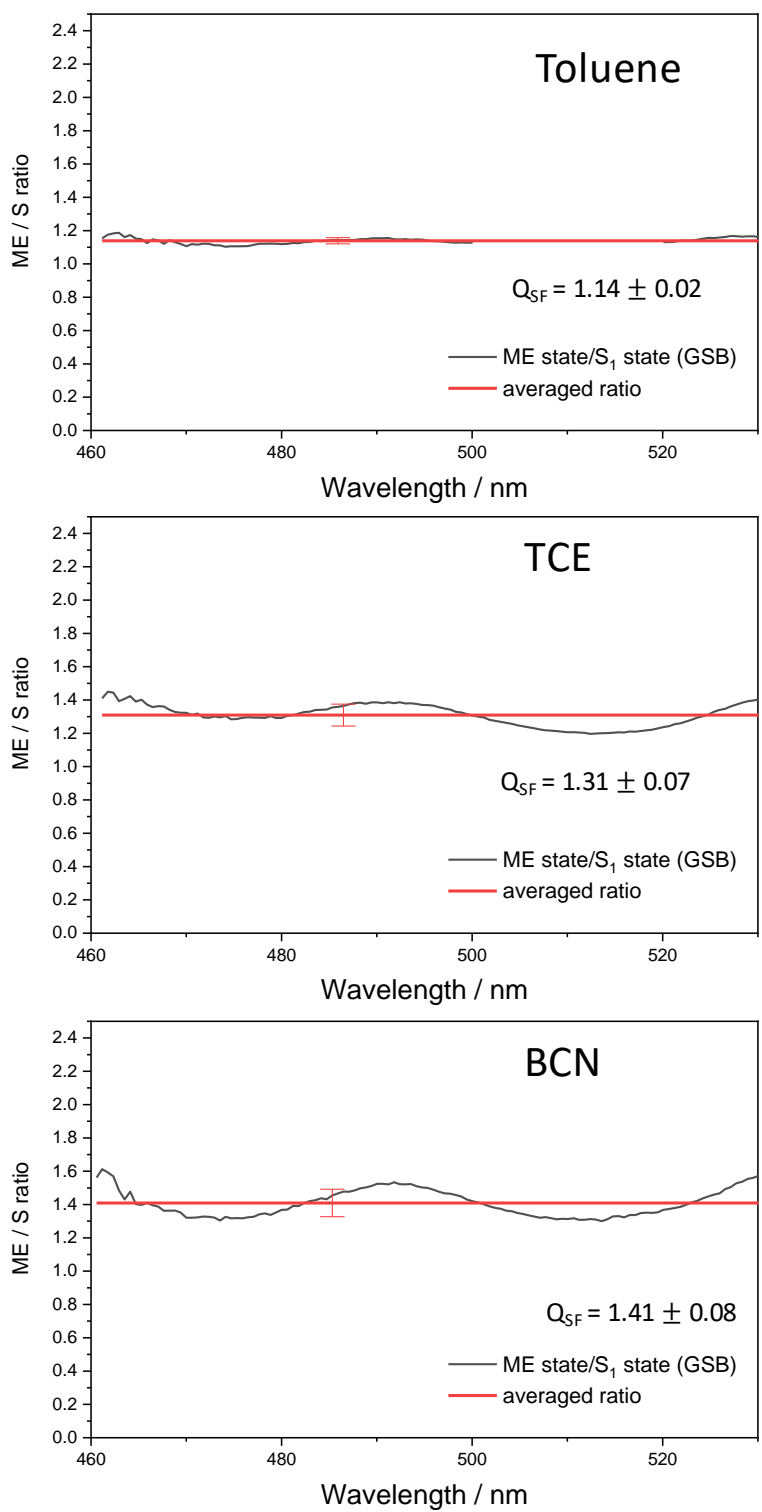

Figure S25. SF yield plot using GSB signal of S $_{1}$ over EAS signal of ME states of Bis-PBI 2. 

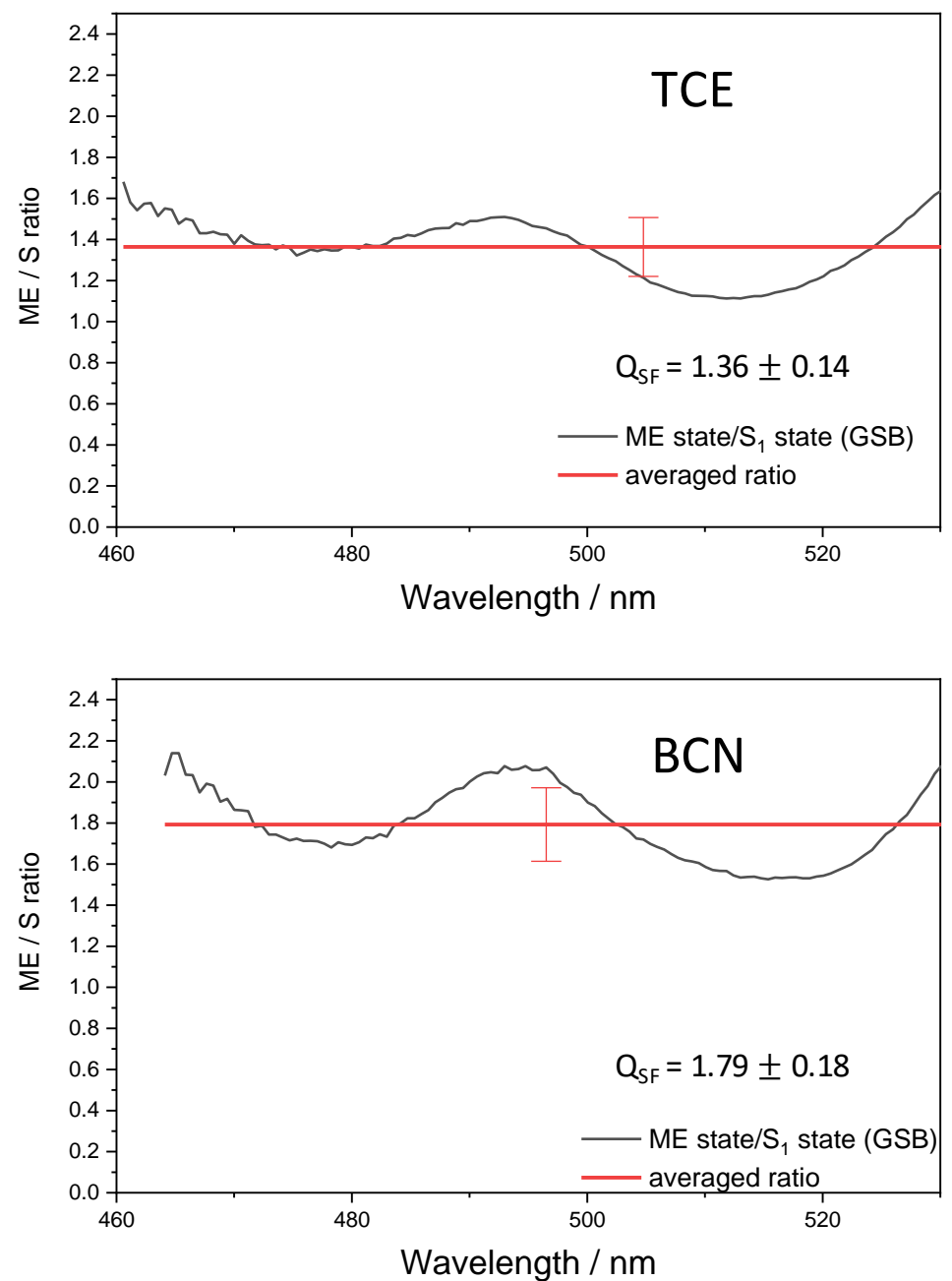

Figure S26. SF yield plot using GSB signal of $S_{1}$ over EAS signal of ME states of Bis-PBI 3. 


\section{1) Sensitization method ${ }^{15}$}

For sensitizer, we used platinum octaethylporphyrin(PtOEP), which has an $100 \%$ of QY $\mathrm{T}_{\mathrm{T}}$. We obtained triplet extinction coefficient of Bis-PBI 2 and Bis-PBI 3 using triplet-triplet energy transfer. Using sensitization method, SF yields exceeding $200 \%$ were observed. The overestimated SF yield is most likely due to the spectral overlap of LE, TT, and CR bands. Furthermore, as the GSB at $550 \mathrm{~nm}$ contains SE signal, the singlet concentration is overestimated. The effect can underestimate the SF yield. (In chlorin solvents, the samples were decomposed.)

The concentration of triplet exciton for Bis-PBIs obtained from $\operatorname{PtOEP}\left(c_{T}^{P B I S}\right)$.

2-1. Triplet energy transfer efficiency.

Photons $/$ Pulse $=\frac{\text { power }}{(\text { rep rate })(\text { energy per photon })}=6.25 \mathrm{E}+13$

Spot volume; V $=$ Area $\bullet$ d $=0.000282743 \mathrm{~L}$

The fraction of light intensity transmitted $\left(\mathrm{I} / \mathrm{I}_{0}\right)$ of PtOEP at $335 \mathrm{~nm}$

$\mathrm{I} / \mathrm{I}_{0}=10^{-\varepsilon_{355 n m}{ }^{C_{\text {P } O E P P}}}=0.075350$ (extinction @ $355 \mathrm{~nm}: 11229.15718 \mathrm{~cm}^{-1} \mathrm{M}^{-1}, \mathrm{c}: 1.1 \cdot 10^{-4} \mathrm{M}$, and L: $1 \mathrm{~cm}$ )

The concentration of triplet exciton for PtOEP:

$c_{T}^{\text {PtOEP }}=\frac{\left(\frac{\text { photons }}{\text { pulse }}\right)\left(1-\frac{I}{I_{0}}\right)}{N_{A} V}=3.39788 \mathrm{E}-07 \mathrm{~mol} / \mathrm{L}$ (in case of Bis-PBI 2 in toluene)

Triplet energy transfer efficiency $(\Phi t)$ was calculated by the ratio of Bis-PBI rise time constant over the sum of the triplet rise and the PtOEP triplet decay constant.

$\Phi=\frac{\frac{1}{8.7 \mu s}}{\frac{1}{8.7 \mu s}+\frac{1}{40 \mu s}}=82.1 \%$

2-2 Triplet concentration

We approximate that triplet exciton of PtOEP transfers to Bis-PBI $\mathbf{n}$ with $82.1 \%$ of efficiency. Therefore, the triplet concentration of Bis-PBI $\mathrm{n}$ is calculated by following Beer's law.

$c_{T}^{P B I S}=4.85412 \cdot 10^{-7} \cdot 0.821=3.98695 \mathrm{E}-07 \mathrm{~mol} / \mathrm{L}$

Using the triplet concentration and ns-TA spectra at $30 \mu \mathrm{s}$, we obtained triplet extinction coefficient depending upon wavelength.

Ex) $\varepsilon_{T}^{580 \mathrm{~nm}}=\frac{(A)_{580 \mathrm{~nm}}}{c_{T}^{P I S}{ }_{A}{ }^{L}}=11931.77227 \mathrm{~mol} / \mathrm{L}$ and $\varepsilon_{T}^{550 \mathrm{~nm}}=\frac{(A)_{550 \mathrm{~mm}}}{c_{T}^{P B I S}{ }_{A}^{L}}=65499.33846 \mathrm{~mol} / \mathrm{L}$

2-3 SF yield

Finally, the SF yield is calculated.

$$
Q_{S F}=\frac{\varepsilon_{S}^{\max }}{\varepsilon_{T}^{x \times n m}} \frac{O D_{M E}}{O D_{S 1}}
$$



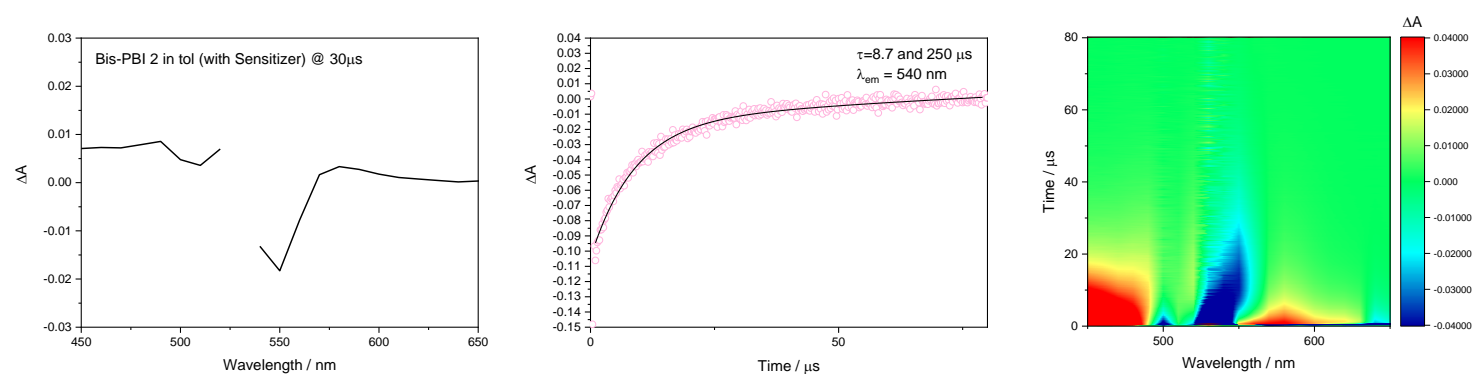

\begin{tabular}{|c|c|c|c|c|c|c|c|}
\hline $\begin{array}{c}\text { Wavelength / } \\
\mathrm{nm}\end{array}$ & 580 & 590 & 600 & 610 & 620 & 630 & $\mathrm{Q}_{\mathrm{SF}}$ \\
\hline $\begin{array}{c}\mathrm{OD}\left(\mathrm{S}_{1}\right) \& \varepsilon_{\text {singlet } @} \\
555 \mathrm{~nm}\end{array}$ & \multicolumn{6}{|c|}{$--0.04504 \& 76700$} & \multirow{4}{*}{$\begin{array}{c}1.32 \\
( \pm 0.49)\end{array}$} \\
\hline$\varepsilon_{\text {triplet }}$ & 11931.77227 & 9889.396836 & 6342.113188 & 3833.933 & 2681.614 & 1565.216 & \\
\hline $\mathrm{OD}(\mathrm{ME})$ & 0.00533 & 0.00985 & \multirow{2}{*}{\multicolumn{4}{|c|}{ Too low extinction coefficient (large error) }} & \\
\hline $\mathrm{Q}_{\mathrm{SF}}$ & -0.760710193 & -1.696146686 & & & & & \\
\hline
\end{tabular}

Figure S27. Summary of sensitization experiments of Bis-PBI 2 in toluene. The top panel shows representative spectra at $30 \mu \mathrm{s}$ (left), decay profile at $540 \mathrm{~nm}$ (middle), and raw TA data (right) of Bis-PBI 2 with PtOEP $\left(1.1^{*} 10^{-4} \mathrm{M}\right)$. Bottom panel indicates the SF yields depending upon the probe wavelength.
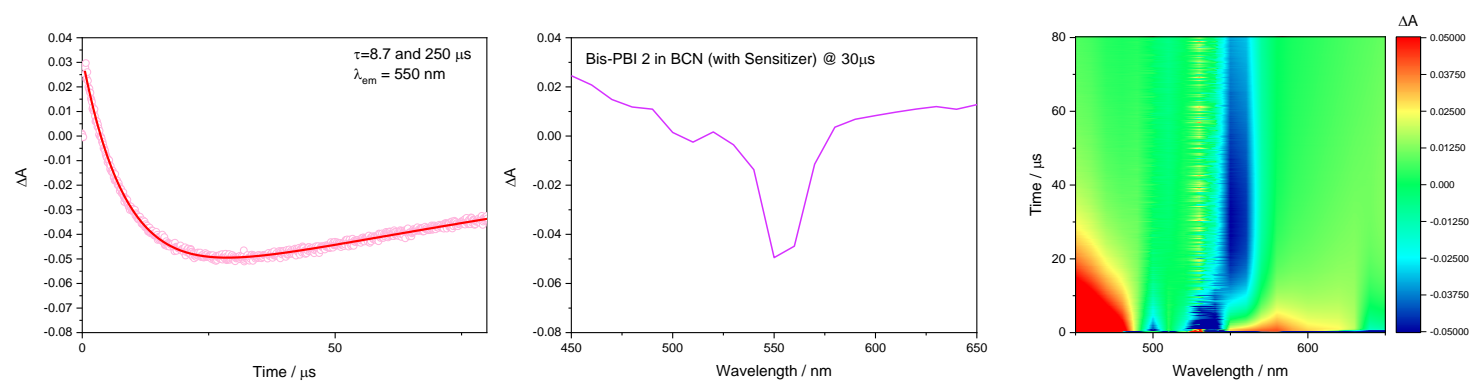

\begin{tabular}{|c|c|c|c|c|c|c|c|}
\hline $\begin{array}{l}\text { Wavelength / } \\
\mathrm{nm}\end{array}$ & 580 & 590 & 600 & 610 & 620 & 630 & $\mathrm{Q}_{\mathrm{SF}}$ \\
\hline $\begin{array}{c}\mathrm{OD}\left(\mathrm{S}_{1}\right) \& \varepsilon_{\text {singlet }} \\
555 \mathrm{~nm}\end{array}$ & \multicolumn{6}{|c|}{$-0.04257 \& 76700$} & \multirow{4}{*}{$\begin{array}{c}2.54 \\
( \pm 0.23)\end{array}$} \\
\hline$\varepsilon_{\text {triplet }}$ & 5608.641496 & 10645.60328 & 12824.16651 & 14987.27893 & 16995.88332 & 18525.51282 & \\
\hline OD (ME) & 0.00722 & 0.01563 & 0.02029 & 0.02357 & 0.02579 & 0.02727 & \\
\hline $\mathrm{Q}_{\mathrm{SF}}$ & -1.221964625 & -2.19594543 & -2.439219991 & -2.83353 & -2.73401 & -2.50826 & \\
\hline
\end{tabular}

Figure S28. Summary of sensitization experiments of Bis-PBI 2 in BCN. The top panel shows representative spectra at $30 \mu \mathrm{s}$ (left), decay profile at $550 \mathrm{~nm}$ (middle), and raw TA data (right) of Bis-PBI 2 with PtOEP $\left(1.1 * 10^{-4} \mathrm{M}\right)$. Bottom panel indicates the SF yields depending upon the probe wavelength. 

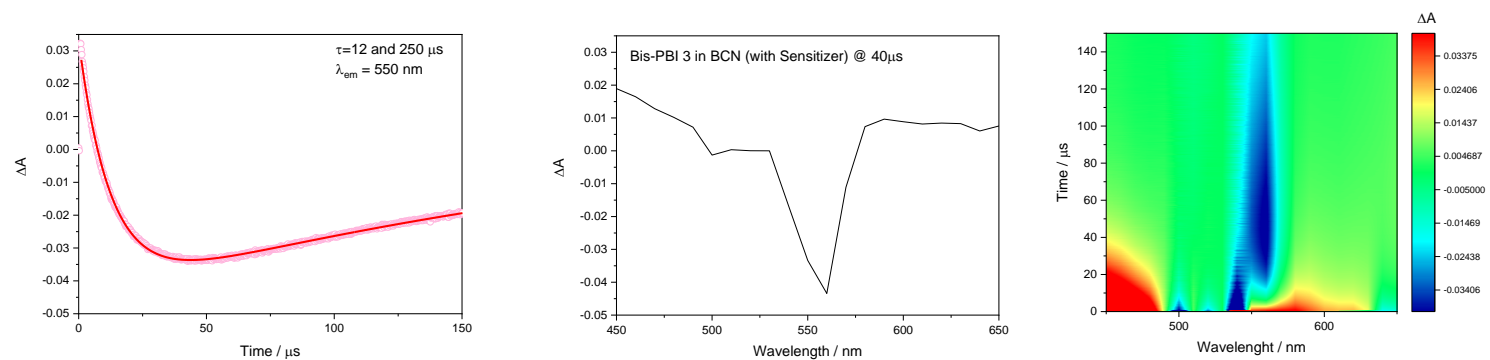

\begin{tabular}{|c|c|c|c|c|c|c|c|}
\hline & 580 & 590 & 600 & 610 & 620 & 630 & $\mathrm{Q}_{\mathrm{SF}}$ \\
\hline $\begin{array}{c}\mathrm{OD}\left(\mathrm{S}_{1}\right) \& \varepsilon_{\text {singlet } @} \\
560 \mathrm{~nm}\end{array}$ & \multicolumn{6}{|c|}{$-0.0366 \& 101500$} & \multirow{4}{*}{$\begin{array}{r}1.28 \\
( \pm 0.50\end{array}$} \\
\hline$\varepsilon_{\text {triplet }}$ & 20892.41048 & 27818.85239 & 25161.36039 & 23182.37699 & 23860.88559 & 23606.44486 & \\
\hline $\mathrm{OD}(\mathrm{ME})$ & 0.00263 & 0.00906 & 0.0117 & 0.01315 & 0.01425 & 0.01534 & \\
\hline $\mathrm{Q}_{\mathrm{SF}}$ & 0.35 & 0.90 & 1.39 & 1.57 & 1.66 & 1.80 & \\
\hline
\end{tabular}

Figure S29. Summary of sensitization experiments of Bis-PBI 3 in BCN. The top panel shows representative spectra at $40 \mu \mathrm{s}$ (left), decay profile at $550 \mathrm{~nm}$ (middle), and raw TA data (right) of Bis-PBI 3 with PtOEP $\left(1.1^{*} 10^{-4} \mathrm{M}\right)$. Bottom panel indicates the SF yields depending upon the probe wavelength.
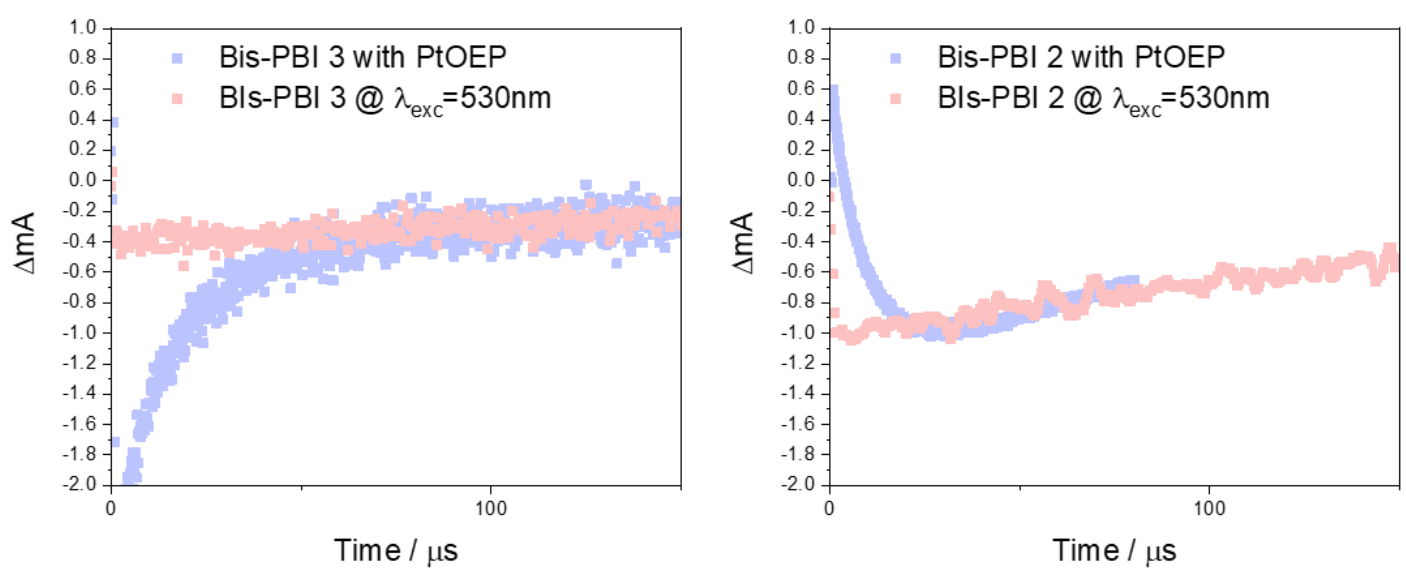

Figure S30. Decay profiles of triplets produced via sensitization by PtOEP in BCN (blue line, scaled) and from direct optical excitation of the Bis-PBI 3 and 2 (red line). (left: Bis-PBI 3 and right: Bis-PBI 2) 


\section{References}

1. Kaufmann, C., Bialas, D., Stolte, M., and Würthner, F. 2018, J. Am. Chem. Soc. 140, 9986-9995.

2. Schanz, R., Kovalenko, S. A., Kharlanov, V., and Ernsting, N. P. Appl. Phys. Lett., 2001, 79, 566-568.

3. Zhao, L., Lustres, J. L. P.; Farztdinov, V., and Ernsting, N. P. Phys. Chem. Chem. Phys., 2005, 7, 1716-1725

4. Zhang, X. -X., Würth, C., Zhao, L., Resch-Genger, U., Ernsting, N. P., and Sajadi, M. Rev. Sci. Instrum., 2011, 82, 063108.

5. Sajadi, M., Quick, M., and Ernsting, N. P. Appl. Phys. Lett. 2013, 103, 173514.

6. Gerecke, M., Bierhance, G., Gutmann, M.; Ernsting, N. P., and Rosspeintner, A. Rev. Sci. Instrum., 2016, 87, 053115.

7. Nakagawa, T., Okamoto, K., Hanada, H., and Katoh, R. Optics Letters, 2016, 7, 1498-1501

8. Koti, A. S. R., and Periasamym N. J. Chem. Phys. 2001, 115, 7094.

9. Beckwith, J. S., Rosspeintner, A., Licari, G., Lunzer, M.; Holzer, B., Fröhlich, J., Vauthey, E. J. Phys. Chem. Lett. 2017, 8, $5878-5883$

10. Mewes, S. A., Plasser, F., Krylov, A., and Dreuw, A. J. Chem. Theory Comput., 2018, 14, 710-725.

11. Farag, M. H., and Krylov, A. I. J. Phys. Chem. C 2018, 122, 25753-25763

12. Kolomeisky, A. B.; Feng, X.; Krylov, A. I. J. Phys. Chem. C 2014, 118, 5188- 5195

13. Feng, X.; Luzanov, A. V.; Krylov, A. I. J. Phys. Chem. Lett. 2013, 4, 3845- 3852.

14. Shao, Y., Gan, Z., Epifanovsky, E., Gilbert, A. T. B., Wormit, M., Kussmann, J., Lange, A. W., Behn, A., Deng, J., Feng, X., Ghosh, D., Goldey, M., Horn, P. R., Jacobson, L. D., Kaliman, I., Khaliullin, R. Z., Kus, T., Landau, A., Liu, J., Proynov, E. I., Rhee, Y. M., Richard, R. M., Rohrdanz, M. A., Steele, R. P., Sundstrom, E. J., Woodcock, H. L., Zimmerman, P. M., Zuev, D., Albrecht, B., Alguire, E., Austin, B., Beran, G. J. O., Bernard, Y. A., Berquist, E., Brandhorst, K., Bravaya, K. B., Brown, S. T., Casanova, D., Chang, C., Chen, Y., Chien, S. H., Closser, K. D., Crittenden, D. L., Diedenhofen, M., DiStasio, R. A., Do, H., Dutoi, A. D., Edgar, R. G., Fatehi, S., Fusti-Molnar, L., Ghysels, A., Golubeva-Zadorozhnaya, A., Gomes, J., HansonHeine, M. W. D., Harbach, P. H. P., Hauser, A. W., Hohenstein, E. G., Holden, Z. C., Jagau, T., Ji, H., Kaduk, B., Khistyaev, K., Kim, J., Kim, J., King, R. A., Klunzinger, P., Kosenkov, D., Kowalczyk, T., Krauter, C. M., Lao, K. U., Laurent, A. D., Lawler, K. V., Levchenko, S. V., Lin, C. Y., Liu, F., Livshits, E., Lochan, R. C., Luenser, A., Manohar, P., Manzer, S. F., Mao, S., Mardirossian, N., Marenich, A. V., Maurer, S. A., Mayhall, N. J., Neuscamman, E., Oana, C. M., Olivares-Amaya, R., O’Neill, D. P., Parkhill, J. A., Perrine, T. M., Peverati, R., Prociuk, A., Rehn, D. R., Rosta, E., Russ, N. J., Sharada, S. M., Sharma, S., Small, D. W., Sodt, A., Stein, T., Stueck, D., Su, Y., Thom, A. J. W., Tsuchimochi, T., Vanovschi, V., Vogt, L., Vydrov, O., Wang, T., Watson, M. A., Wenzel, J., White, A., Williams, C. F., Yang, J., Yeganeh, S., Yost, S. R., You, Z., Zhang, I. Y., Zhang, X., Zhao, Y., Brooks, B. R., Chan, G. K. L., Chipman, D. M., Cramer, C. J., Goddard, W. A., Gordon, M. S., Hehre, W. J., Klamt, A., Schaefer, H. F., Schmidt, M. W., Sherrill, C. D., Truhlar, D. G., Warshel, A., Xu, X., Aspuru-Guzik, A., Baer, R., Bell, A. T., Besley, N. A., Chai, J., Dreuw, A., Dunietz, B. D., Furlani, T. R., Gwaltney, S. R., Hsu, C., Jung, Y., Kong, J., Lambrecht, D. S., Liang, W., Ochsenfeld, C., Rassolov, V. A., Slipchenko, L. V., Subotnik, J. E., Van Voorhis, T., Herbert, J. M., Krylov, A. I., Gill, P. M. W., Head-Gordon, M. Mol. Phys. 2015, 113, 184-215.

15. Liu, H., Wang, Z., Wang, X., Shen, L., Zhang, C., Xiao, M., and Li, X. J. Mater. Chen. C 2018, 6, 3245-3253.

16. Koch, M.; Myahkostupov, M.; Oblinsky, D. G.; Wang, S.; Garakyaraghi, S.; Castellano, F. N.; Scholes, G. D. J. Am. Chem. Soc. 2017, 139, 5530-5537. 